Chapman University

Chapman University Digital Commons

Food Science (MS) Theses

Dissertations and Theses

Summer 8-2020

\title{
Elucidating the Relation between Human Milk Fatty Acids, Extracellular Vesicles, and Infant Developmental Outcomes in the First Year of Life
}

Diana Bickmore

Chapman University, bickmore@chapman.edu

Follow this and additional works at: https://digitalcommons.chapman.edu/food_science_theses

Part of the Human and Clinical Nutrition Commons, Nutritional Epidemiology Commons, and the Other Food Science Commons

\section{Recommended Citation}

Bickmore, D.C. (2020). Elucidating the relation between human milk fatty acids, extracellular vesicles, and infant developmental outcomes in the first year of life. Master's Thesis, Chapman University.

https://doi.org/10.36837/chapman.000169

This Thesis is brought to you for free and open access by the Dissertations and Theses at Chapman University Digital Commons. It has been accepted for inclusion in Food Science (MS) Theses by an authorized administrator of Chapman University Digital Commons. For more information, please contact laughtin@chapman.edu. 
Elucidating the relation between human milk fatty acids, extracellular vesicles, and infant developmental outcomes in the first year of life

\author{
A Thesis by \\ Diana Claire Bickmore \\ Chapman University \\ Orange, CA \\ Schmid College of Science and Technology \\ Submitted in partial fulfillment of the requirements for the degree of \\ Master of Science in Food Science
}

August, 2020

Committee in charge:

John Miklavcic, Ph.D., Advisor

Laura Glynn, Ph.D.

Anuradha Prakash, Ph.D. 
The thesis of Diana Claire Bickmore is approved
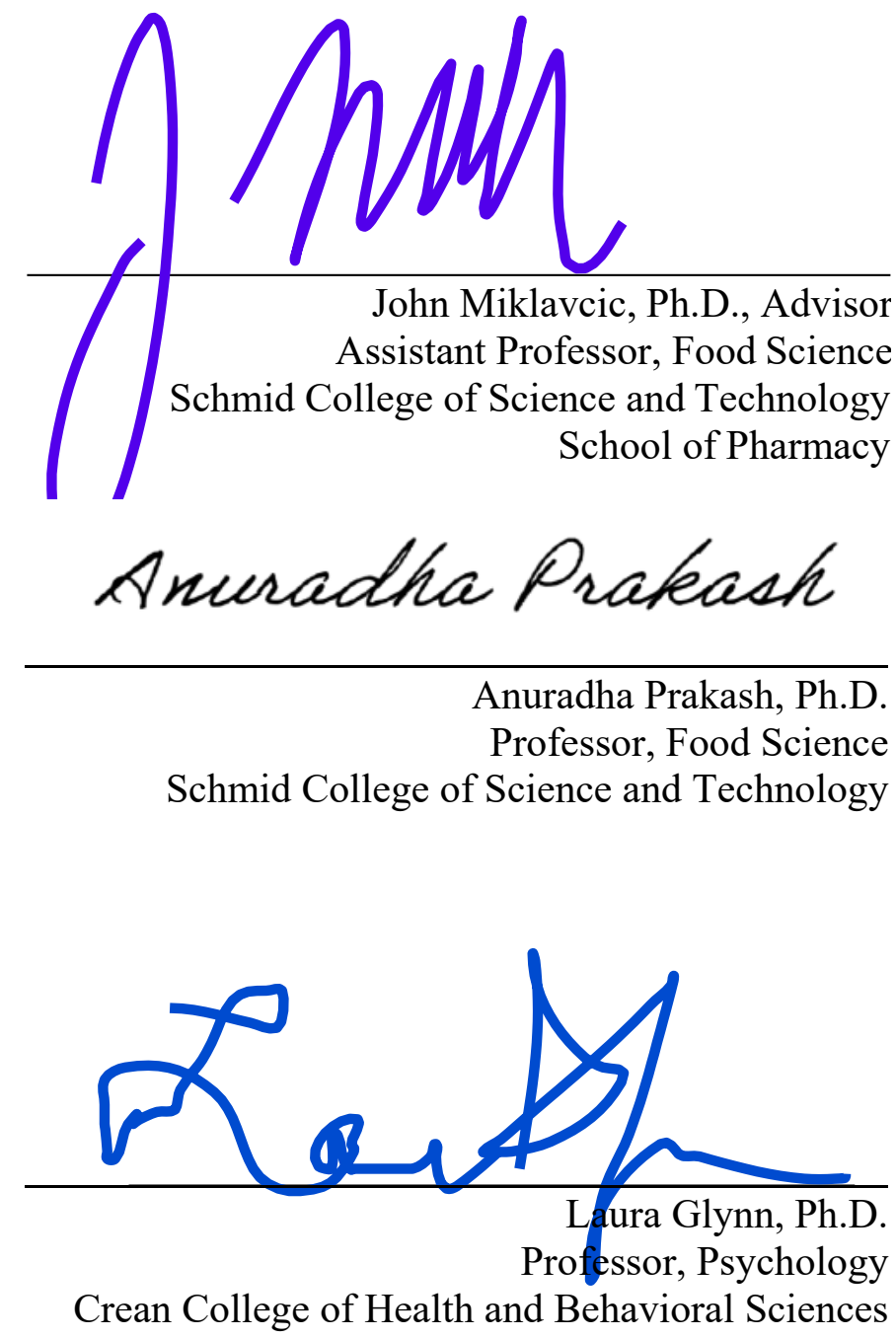

May, 2020 
Elucidating the relation between human milk fatty acids, extracellular vesicles, and infant health outcomes in the first year of life

Copyright (C) 2020

by Diana Claire Bickmore 


\section{ACKNOWLEDGMENTS}

I would like to thank Dr. Miklavcic for the many research opportunities that I have benefited from during my time at Chapman, the members of my thesis committee for taking the time oversee the completion of this project, and Dr. Were for providing extensive feedback on my thesis proposal. I would also like to thank Robyne Kelly, Marina Barsoum, my program peers, friends, and parents for supporting and encouraging me throughout the past two years. 


\section{ABSTRACT \\ Elucidating the relation between human milk fatty acids, extracellular vesicles, and infant health outcomes in the first year of life}

by Diana Claire Bickmore

For most infants, human milk is the recommended source of nutrition. Improved developmental outcomes have been reported in infants that consume human milk compared to infant formula. Essential fatty acids (EFAs) confer health benefits. However, EFAs cannot be synthesized by the body, and therefore must be consumed in diet. Extracellular vesicles (EVs) are nanoparticles containing a lipid bilayer membrane and are present in human milk. Methods of EV isolation such as ultracentrifugation (UC) may not be feasible for the study of EVs in human milk due to the need for large sample volume, which may not be available. The objectives of this research were therefore to investigate i) if the concentration of essential fatty acids in milk correlated to infant developmental outcomes reported by the Bayley Scales of Infant Development (BSID-III) and ii) if an EV isolation method using a precipitation method could be optimized for isolation of EVs in a low volume of human milk. Human milk samples were obtained two weeks postpartum $(\mathrm{n}=70)$, and corresponding developmental data was obtained. Total fatty acids from milk samples were quantified by Gas Chromatography-Mass Spectrometry (GC-MS). Electron microscopy, nanoparticle tracking analysis, and semi-quantitative antibody array were conducted to confirm isolation of human milk EVs. Count, size, protein content, and fatty acid quantification of EVs were also determined. Arachidonic acid was inversely correlated with Cognitive Composite scores at 12 months of age $(p<0.05)$. The EV isolation technique yielded $8.9 \times 10^{9} \pm 1.1 \times 10^{9} \mathrm{EV}$ particles $/ \mathrm{mL}$ of human milk, and meets the Minimal Information for 
Studies of Extracellular Vesicles (MISEV) guidelines. Validation of an EV isolation protocol for small volumes of human milk will facilitate research in this growing area. It can be concluded that bioactive components of human milk are related to infant development, and future research on fatty acids and EVs and human milk is warranted to assist nutritional recommendations for breastfeeding mothers and manufacturers of commercial infant formula. 


\section{TABLE OF CONTENTS}

1. BIOACTIVE COMPONENTS IN HUMAN MILK: A LITERATURE REVIEW AND INTRODUCTION TO THE PRESENT RESEARCH

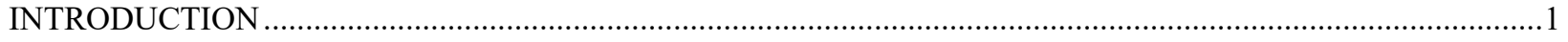

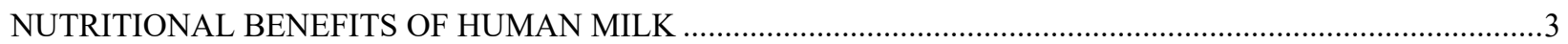

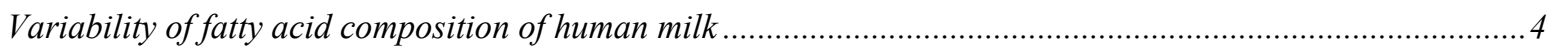

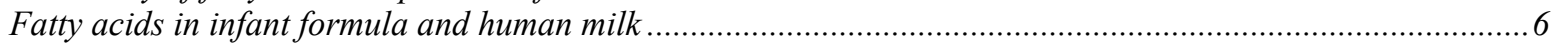

Standardized health and developmental outcomes in relation to human milk .................................................9

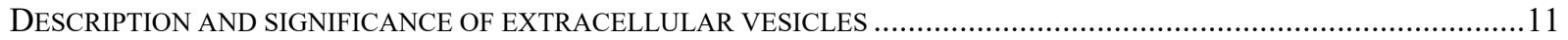

Lipids and fatty acids in extracellular vesicles ...................................................................................

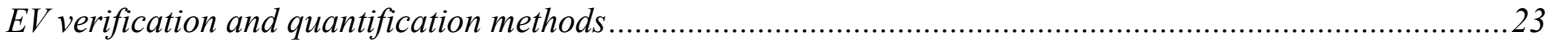

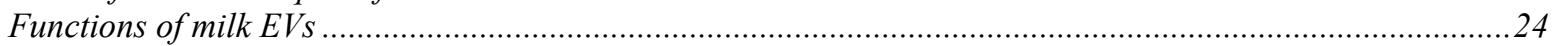

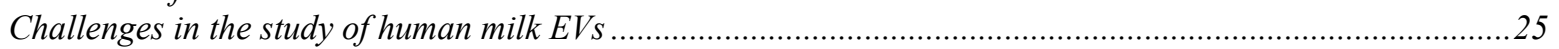

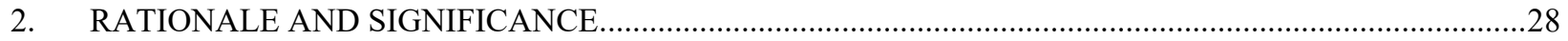

3. THE RELATION BETWEEN THE FATTY ACID COMPOSITION OF HUMAN MILK AND BSID-III

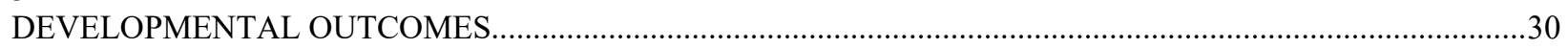

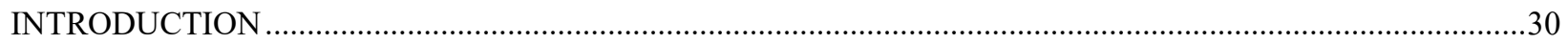

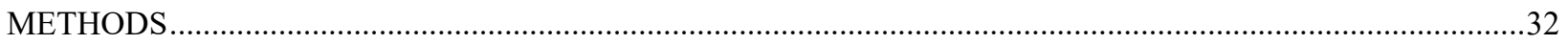

ACQUISITION OF HUMAN MILK AND DEVELOPMENTAL DATA .............................................................................32

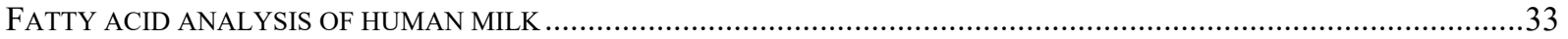

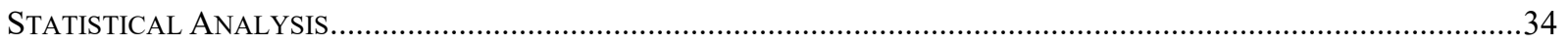

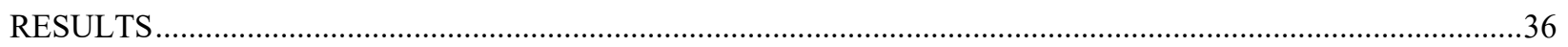

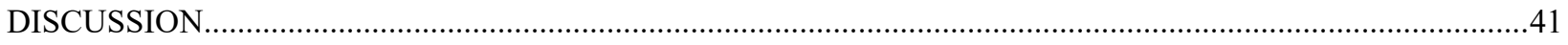

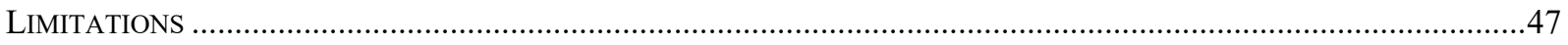

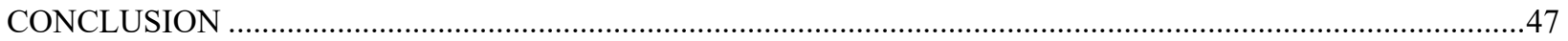

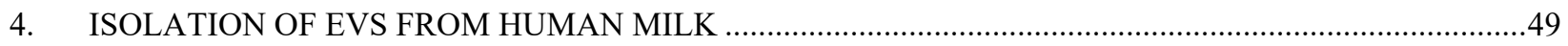

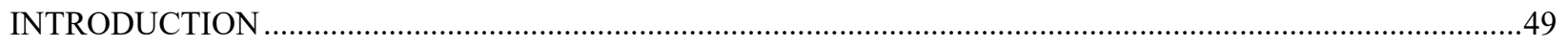

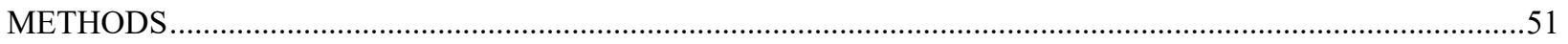

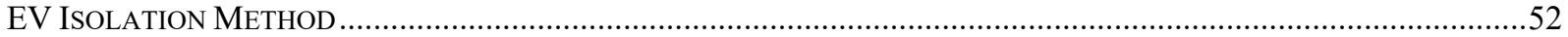

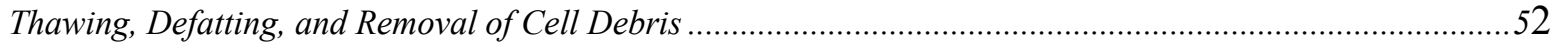

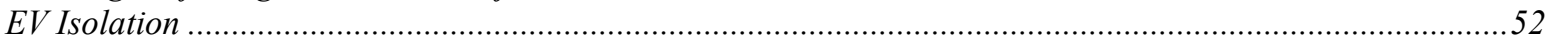

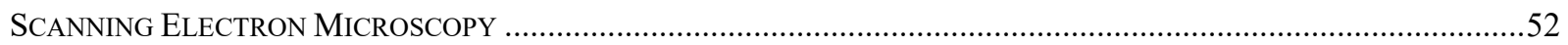

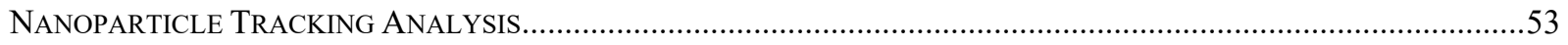

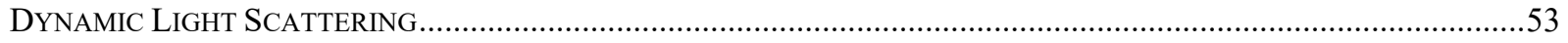

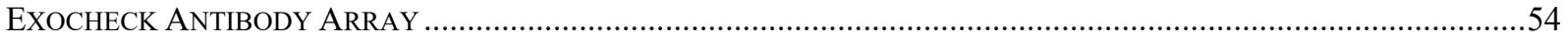

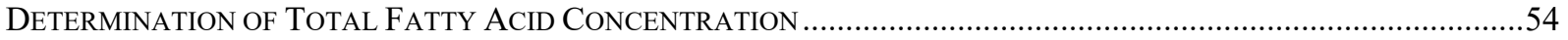

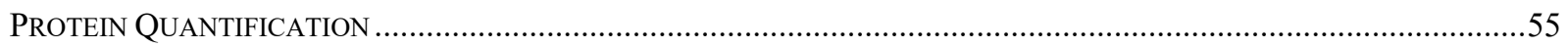

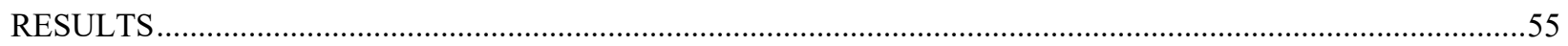

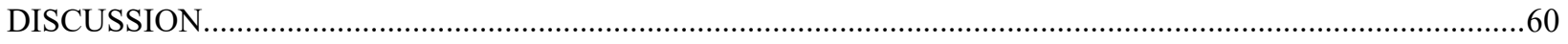

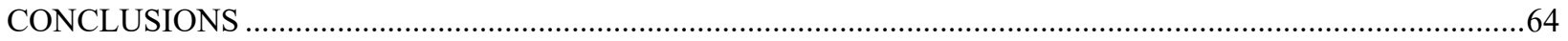


5. FATTY ACIDS AND EVS IN HUMAN MILK: GLOBAL DISCUSSION, CONCLUSIONS, AND FUTURE

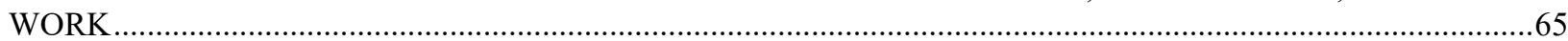

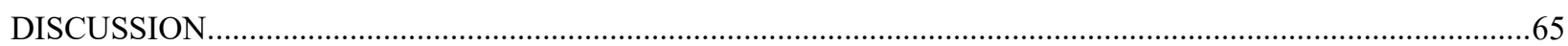

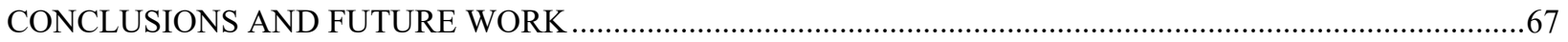

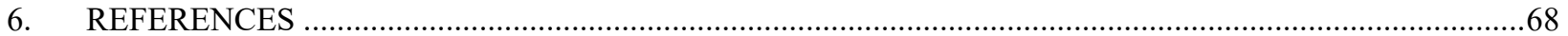




\section{LIST OF TABLES}

Table 1-1. Summary of lipids and fatty acids in EVs in the literature...................................16

Table 1-2. Summary of the applications of different exosome isolation techniques............18

Table 3-1. Maternal and demographic information and categorical level of education for the 70

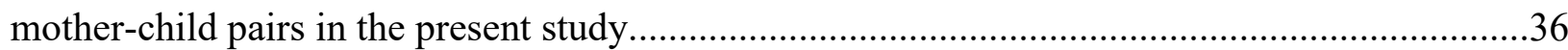

Table 3-2. Descriptive statistics for the Bayley Cognitive, Motor, and Language Composite

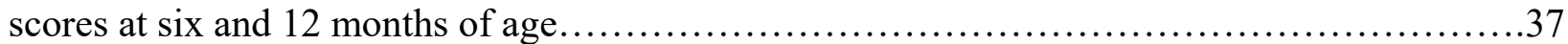

Table 3-3. Descriptive statistics for the select fatty acid composition of human milk $(n=70)$

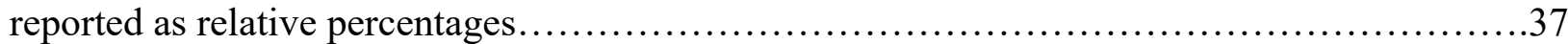

Table 3-4. Linear regression analysis for fatty acids and BSID-III scores......................38

Table 3-5. Regression analysis of ARA and Bayley Cognitive Composite and Language

Composite scores at 12 months of age after adjusting for covariates .39

Table 3-6. Linear regression model of ARA in relation to Bayley Cognitive Composite scores at 12 months of age after incorporating the following modifier: Percentage of infant diet comprised of human milk at six months.

Table 3-7. Average fatty acid content of human milk at one month postpartum in mothers from Northwestern Spain compared to the fatty acid content of mothers in the present study

Table 4-1. Protein concentration of EVs isolated from human milk of 10 different participants measured in duplicate 


\section{LIST OF FIGURES}

Figure 1-1. Chemical structures of linoleic acid (a) and alpha-linolenic acid (b), the two precursor essential fatty acids..................................................

Figure 1-2. Formation and release of exosomes from cells...............................12

Figure 1-3. Comparison of UC with a commercial kit for isolation of EVs in human milk.......21

Figure 4-1. Workflow schematic for EV isolation using a precipitation-based isolation

method

Figure 4-2. Image of EVs obtained by SEM from participant.

Figure 4-3. Diameter and concentration of human milk EVs from pooled human donor milk

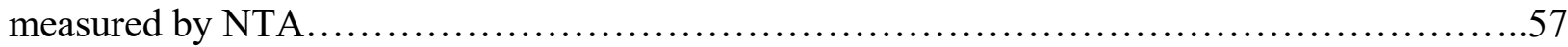

Figure 4-4. Average diameter of EVs from pooled human donor milk measured for 10

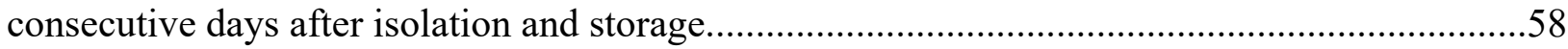

Figure 4-5. Antibody array of human milk EVs from participant..........................58

Figure 4-6. Antibody array of human milk EVs from participant........................59 


\section{LIST OF ABBREVIATIONS}

ARA: Arachidonic acid

DHA: Docosahexaenoic Acid

EFA: Essential Fatty Acids

EV(s): Extracellular vesicle

FADS: Fatty acid dehydrogenase

LCPUFA: long-chain polyunsaturated FAs

MFGM: Milk fat globule membrane

MV: Microvesicle

NEC: Necrotizing enterocolitis

PUFA: Polyunsaturated fatty acid

PBS: Phosphate-buffered saline 


\section{Bioactive Components in Human Milk: A literature review and introduction to the present research}

\section{Introduction}

In 2015 only about $25 \%$ of babies in the United States were exclusively breastfed for the first 6 months of life. The American Academy of Pediatrics recommends that infants should be exclusively breastfeed for the first 6 months of life (CDC, 2018). Because human milk is the recommended source of nutrition for infants, a greater understanding of its composition is crucial to public health. There is therefore a critical need to explore the associations between infant health outcomes, and bioactive components in human milk. This may facilitate nutritional recommendations for breastfeeding mothers in the perinatal period, and inform the nutritional composition of commercial infant formulas made to mimic the biological properties of human milk and human milk fortifiers designed to optimize human milk. The present study will define infants as children younger than one year of age (WHO, 2013), and newborns as children under one month of age.

Fatty acids in human milk have been linked to improved infant health and developmental outcomes. The consumption of essential fatty acids (EFA) by infants, such as docosahexaenoic acid (DHA) and eicosopentanoic acid (EPA) has been correlated to superior visual acuity and cognitive development (Makrides, Simmer, Goggin, \& Gibson, 1993), and infants consuming infant formula fortified with DHA and arachidonic acid (ARA) scored closer to infants fed human milk (Lien, Richard, \& Hoffman, 2018). Although human milk is the recommended source of nutrition for the first six months of life, the relation between bioactive compounds, such as essential fatty acids present in human milk and standardized developmental outcomes in the absence of confounding variables associated with breastfeeding remains unclear. 
Extracellular vesicles (EV) are a type of bioactive component present in human milk that may confer health benefits to newborns and infants. EVs are produced by nearly every type of mammalian cell for cell to cell communication (De la Torre Gomez, Goreham, Serra, Nann, \& Kussman, 2018). EVs are composed of proteins, nucleic acids, and lipids. Lipids and fatty acids are crucial components of extracellular membranes, and specific lipids and fatty-acid containing lipids such as sphingomyelin and cholesterol have been found in higher concentrations in EVs compared to the cell from which it was derived (Llorente et al., 2013). EVs in human milk have been found to mediate immune and inflammatory responses and the promotion of epithelial growth in the intestine. (De la Torre Gomez et al., 2018; Hock et al., 2017b).

This gap in knowledge may exist partly due to the difficulty of isolating EVs. Although ultracentrifugation (UC) is the "gold standard" technique for the isolation of EVs, it requires sample volumes in excess of several $\mathrm{mL}$, and may result in the co-isolation of contaminants like albumins and immunoglobulins (Caradec et al., 2014). Using a large sample volume for isolation of EVs from human milk is often not feasible, as the milk is needed to feed the infant, and mothers may not lactate sufficient excess volumes to be collected for research. The necessity of large sample volumes of human milk for research purposes relegates researchers to only studying mature milk. This hinders the study of variation over i) early, transitional and mature milk production periods, ii) course of lactation (fore vs. hind milk), and iii) time-of-day.

The overarching goals of this project were to correlate the fatty acid composition of human milk and human milk EVs to infant health and development (Cognitive, Behavioral, and Motor) outcomes reported by the Bayley Scales of Infant Development (BSID-III). It was hypothesized that bioactive components in human milk are related to developmental outcomes in infants. To investigate this hypothesis, essential fatty acids were correlated to BSID-III 
outcomes. Determining the relation between the fatty acid content of EVs in human milk and infant developmental outcomes was not feasible due to the lack of a suitable EV isolation method. Therefore, the second objective became to optimize an EV isolation method for low $(<2$ $\mathrm{mL}$ ) volumes of human milk to facilitate the study of EV composition and infant health and developmental outcomes in future research.

\section{Review of Literature}

\section{Nutritional benefits of human milk}

For most infants, human milk provides the best source of nutrition and benefits the health of both mothers and infants, reducing risk for short and long-term health conditions include asthma, obesity, Type II diabetes, ear and respiratory infections, and gastrointestinal infections (CDC, 2018). Infants consuming human milk benefit epidemiologically from protection against illness and infection due to specific and non-specific immune factors found in human milk (Oddy, 2002); protective components in milk such as secretory IgA antibodies and lactoferrin confer immunity against common infections to breastfed infants (Hanson et al., 2003). The benefits of consuming human milk may also extend beyond infancy. (Wilson et al., 1998) investigated the correlation between exclusive consumption of human milk in infancy and childhood respiratory illness, growth, and body composition, and found that infants who were exclusively fed human milk for 15 weeks (compared to infants partially fed human milk for 15 weeks and formula fed infants) experienced lower incidences of respiratory illnesses and wheezing during childhood, as well as lower percentages of body fat and weight, indicating less risk of childhood obesity. Consumption of human milk has even been correlated with lower rates of AttentionDeficit/Hyperactivity Disorder (ADHD) in children (Mimouni-Bloch et al., 2013). Despite the multitude of short and long-term benefits associated with consuming human milk, the majority of 
infants do not consume human milk exclusively for as long as recommended by the World Health Organization and American Academy of Pediatrics, with only 1 in 4 exclusively breastfed for 6 months (CDC, 2018).

\section{Variability of fatty acid composition of human milk}

Human milk is widely considered more nutritious for infants than infant formula.

Studying the composition of human milk can therefore help create more nutritious infant formula products. The fatty acid composition of human milk was examined using gas-liquid chromatography and spectrophotometry (Insull \& Ahrens, 1959). It was found that human milk fatty acids consisted of: oleic (29\%), palmitic (21\%), myristic (9\%), linoleic (7\%), stearic (7\%), lauric (7\%), isooleic (7\%), palmitoleic (2\%), and other species in minor quantities. More recently, meta-analyses have compared the composition of human milk fatty acids at similar points of lactation worldwide (Barreiro, Díaz-Bao, Cepeda, Regal, \& Fente, 2018), and fatty acids present in smaller amounts have been quantified. Comparing 21 studies of the fatty acid composition of human milk worldwide to the composition of human milk in Galicia, Spain, it was found that worldwide, fatty acid profiles of human milk were similar, with the exception of several Asian countries (Barreiro et al., 2018). Women in Asian countries where consumption of marine products is high had elevated levels of DHA in milk. While maternal diet is a well-known factor that alters the fatty acid composition of human milk, other variables that may influence the fatty acid composition of human milk have also been investigated. In a study of 71 Australian infants exclusively consuming human milk, it was suggested that the average fat content was independent of the gender of the infant, and that the mean fat content of milk was significantly $(p<0.001)$ related to the time of day, with the mean fat content of the milk collected in the day and evening ( $42.8 \pm 9.1$ and $43.2 \pm 9.1 \mathrm{~g} / \mathrm{L}$, respectively) being higher than the milk collected in 
the morning and at night $(37.1 \pm 10.1$ and $37.2 \pm 10.3 \mathrm{~g} / \mathrm{L}$, respectively), which was likely due to more milk being removed during the day and evening from feedings (Kent et al., 2006).

\section{Essential fatty acids, and fatty acid desaturase}

Omega-3 and omega-6 are classes of polyunsaturated fatty acids (PUFAs) consisting of at least one cis-double bond (Food and Nutrition Board, 2002). In omega-6 fatty acids, the last double bond is situated between the sixth and seventh carbon atom from the methyl end of the fatty acid, and in omega-3 fatty acids, the last double bond is situated between the third and fourth carbon atom from the methyl end of the fatty acid. The two precursors to long chain polyunsaturated fatty acids (LC-UPUFAs) are linoleic acid (LA) and alpha-linolenic acid (ALA) (Figure 1-1). LA (18:2n-6) and ALA (18:3n-3) have no known biological activity. LA and ALA are essential because they cannot be synthesized by humans, meaning they must be consumed in diet to prevent nutritional deficiency. Although humans are able to synthesize saturated fatty acids and monounsaturated fatty acids from acetyl coenzymeA, humans are not able to synthesize EFA because they do not have the enzymes needed to add a cis-double bond at the n3 or n-6 position. Long-chain omega-3 and omega-3 products docosahexaeonoic acid (DHA) and arachidonic acid (AA) respectively can be synthesized from their ALA and LA precursors respectively (Tressou, Buaud, Simon, Pasteau, \& Guesnet, 2019), albeit with very low efficiency.

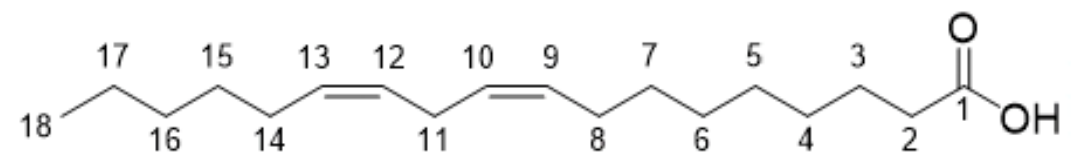

a.) 


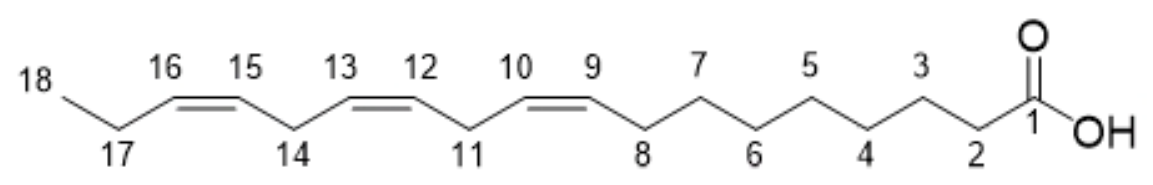

b.)

Figure 1-1. Chemical structures of linoleic acid (a) and alpha-linolenic acid (b), the two precursor essential fatty acids. Created with ChemDraw 18.

Deficiencies in EFA have been associated with decreased growth in children and infants, greater susceptibility to infections, and slower wound healing (Jeppesen, Høy, \& Mortensen, 1998). Omega-3 and omega-6 PUFAs are structural components of plasma membranes, and can influence membrane properties including fluidity, permeability, and activity of membrane-bound enzymes (Nakamura \& Nara, 2004). However, there is inter-individual variability in the metabolism of fatty acids. The FADS-1 and FADS-2 enzymes modulate the synthesis of ARA and DHA from LA and ALA. Polymorphisms in the FADS-1 and FADS-2 genes encode the enzymes that alter the production and thus concentration of LC-PUFAs (Lattka, Illig, Heinrich, $\&$ Koletzko, 2009) for consumption in human milk. Therefore, fatty acid content of infants and newborns consuming human milk as well as maternal FADS genotype may impact health outcomes of newborns and infants.

\section{Fatty acids in infant formula and human milk}

DHA and ARA are conditionally essential fatty acids since (Larque, Demmelmair, \& Koletzko, 2002) infants are able to synthesize DHA from ALA and ARA from LA. However, infants are not able to synthesize sufficient amounts to maintain cellular and plasma DHA concentrations without consuming additional DHA from diet (Larque et al., 2002). When infants 
consume pre-formed ARA and DHA in human milk, they exhibit higher plasma and tissue phospholipid levels than babies that consume infant formula. It has therefore been proposed that infant formulas be fortified with DHA so the cellular and plasma DHA levels of formula fedinfants could reach those of infants consuming human milk (Larque et al, 2002). ARA is typically less varied in human milk than DHA (Carlson \& Colombo, 2016).

Like DHA, ARA has been associated with infant growth and development. Higher levels of erythrocyte phospholipid ARA concentrations in infants were correlated to increased growth (weight and length) at 2, 4, 6.5, and 9 months of age (Carlson, Werkman, Peeples, Cooke, \& Tolley, 1993), and infants consuming human milk and infants consuming formula supplemented with DHA and ARA were found to have better visual acuity at 2 months of age (Carlson, Ford, Werkman, Peeples, \& Koo, 1996). Although infants have the ability to synthesize ARA and DHA from the precursors, infants consuming human milk with lower concentrations of ARA and DHA, and infants consuming infant formula that is not fortified with ARA and DHA may still experience deleterious health impacts due to the inherent inefficiency of ARA and DHA conversion. The negative implications associated with ARA and DHA deficiency during infancy warrant further consideration for infants fed human milk and formula.

\section{Infant health in relation to maternal diet}

The milk composition of indigenous women in Bolivia was examined. Milk samples of Bolivian women were compared to samples from women in the United States at corresponding lactational stages to compare the fatty acids (M. Martin et al., 2012). It was found that the percentages of ARA, DHA, total n-3 and total n-6 LCPUFAs were significantly greater in the indigenous women. This was likely due to their traditional diet including higher consumption of wild game, freshwater fish, and a limited consumption of processed foods and oil. These findings 
have important implications for maternal nutrition. If the fatty acid composition of human milk can be easily altered by diet, women can consume diets that would result in a favorable fatty acid composition of milk. Alternatively, human milk fortifiers could be utilized to compensate for dietary deficiencies.

Maternal nutrition is important for breastfeeding mothers, since maternal diet impacts the composition of human milk. Perinatal nutrition has acute and chronic impacts on immunity and metabolism in newborns, babies, infants and children (De la Torre Gomez et al., 2018). Maternal protein intake is positively correlated with lipid concentration of human milk after 16 weeks postpartum (Nommsen, Lovelady, Heinig, Lönnerdal, \& Dewey, 1991). Additionally, in a randomized control trial in which mothers were assigned to either a placebo or DHAsupplemented group, the DHA concentrations in the milk of the supplemented group significantly ( $<<0.05)$ increased (Valentine et al., 2012) over time relative to the placebo group. This evidence reaffirms that the presence of specific fatty acids in maternal diet alters the fatty acid composition of human milk, and that maternal nutrition impacts the composition of human milk.

\section{Standardized health and behavioral tests for infants}

\section{The Bayley Scales of Infant Development}

The BSID-III is an individually administered test to measure developmental functioning of infants and toddlers. The test identifies developmental delay and accelerated developmental trajectories and tracks a child's developmental progress. The BSID-III can be administered to children between 1 and 42 months of age. The BSID-III is comprised of Cognitive, Language, Motor, Social-Emotional, and Adaptive Behavior Scales (Bayley, 2006), and includes activities 
such as pretend play, novelty preference, habituation, and number ordering. Based on the composite scores in the various scales, the test identifies signs of developmental delay and advanced developmental trajectories. To that end, the primary goal of the Bayley-III is simply to identify whether or not children are experiencing developmental delay, not to provide a specific diagnosis for a disorder (Albers \& Grieve, 2007). The BSID-III was designed to be simpler with respect to the administration of the test. Scoring is less ambiguous than in previous editions, as the only possible scores recorded by examiners trained in developmental interpretation and assessment are 0 and 1 (no credit and credit respectively). Typically a caregiver remains present in the room during administration of the BSID-III to ensure accurate representations of the infant's optimal performance and prevent negative behavioral reactions that may stem from separation. The chronological age of the child being examined corresponds to a designated starting point for Cognitive, Motor, and Language scales, and scoring is discontinued if five consecutive items are given no credit (Albers \& Grieve, 2007).

Of the five scales that comprise the BSID-III, the following three are administered by the clinician: Cognitive, Motor, and Language. Specifically, the Cognitive scale assesses play skills, information processing related to attention to novelty and memory, and problem-solving, counting, and numerical skills. The Language Scale assesses receptive and expressive language to examine communication skills, and the Motor Scale examines both Fine and Gross Motor skills (Bayley, 2006).

\section{Standardized health and developmental outcomes in relation to human milk}

Human milk is considered the optimal source of infant nutrition for newborns. However, there is a lack of information correlating standardized measures of health and developmental outcomes to the composition of human milk. There is also a lack of information correlating 
specific bioactive components of human milk, such as fatty acids, to these outcomes. Although human milk consumption has been correlated with increased cognitive functioning in infants, many of the correlations found between consumption of human milk and superior cognitive functioning may be confounded by other factors associated with breastfeeding such as maternal socioeconomic class and intelligence quotient (IQ) (Walfisch, Sermer, Cressman, \& Koren, 2013).

Although specific fatty acids, such as DHA and AA, have been associated with cognitive benefits when used as ingredients in infant formulas (Larque et al., 2002), the benefits associated with bioactive components of human milk are also often limited by confounding factors. Various experiments have only been designed to correlate newborns fed human milk to those fed infant formula with particular health and behavioral outcomes, meaning the actual composition of the human milk that breastfed infants consume is not taken into account.

In a study of 771 low birthweight infants, babies whose mothers provided human milk instead of formula exhibited greater mean Bayley developmental index scores even after adjusting for demographic and perinatal factors (Morley, Cole, Powell, \& Lucas, 1988). Likewise, after adjusting for the several confounding factors, including maternal age, intelligence, education, and smoking status during pregnancy, it was found that infants fed human milk scored higher on the Bayley mental development index (MDI) at 13 months of age if they had been fed human milk for six months after birth instead of fewer than three months (Angelsen, Vik, Jacobsen, \& Bakketeig, 2001). The present study will build upon the prior knowledge correlating consumption of human milk to particular health and behavior outcomes by correlating specific bioactive components of human milk, such as fatty acids, instead of human milk as a whole, to health and behavioral outcomes. 
The BSID-III was used to compare infants fed formula exclusively and infants fed both human milk and formula, as well as infants fed human milk exclusively in bottles versus human milk directly from the breast (Pang et al., 2019). It was found that infants who were bottle-fed human milk exhibited significantly better Cognitive scores on the BSID-III at 2 years than infants only fed infant formula. Interestingly, compared to the infants who were bottle-fed human milk, infants who consumed human milk by breastfeeding achieved higher scores on several memory tasks. Thus, confounding factors such as the discrepancy in the BSID-III scores between infants that were breastfed and bottle-fed human milk emphasize the importance of relating specific components found in human milk to test outcomes, and highlight that results from infants consuming human milk administered differently should not be considered analogous.

\section{Description and significance of extracellular vesicles}

EVs were first described in 1983 as vesicles secreted by red blood cells, but became of interest to immunologists when it was discovered (Raposo et al., 1996) that cells of the immune system could secrete exosomes when multivesicular bodies (MVBs) fused with the plasma membrane (Bobrie, Colombo, Raposo, \& Théry, 2011). The term "exosome" was proposed to refer to the extracellular vesicles that originated from intraendosomal regions of mammalian cells (Johnstone, Adam, Hammond, Orr, \& Turbide 1987). 


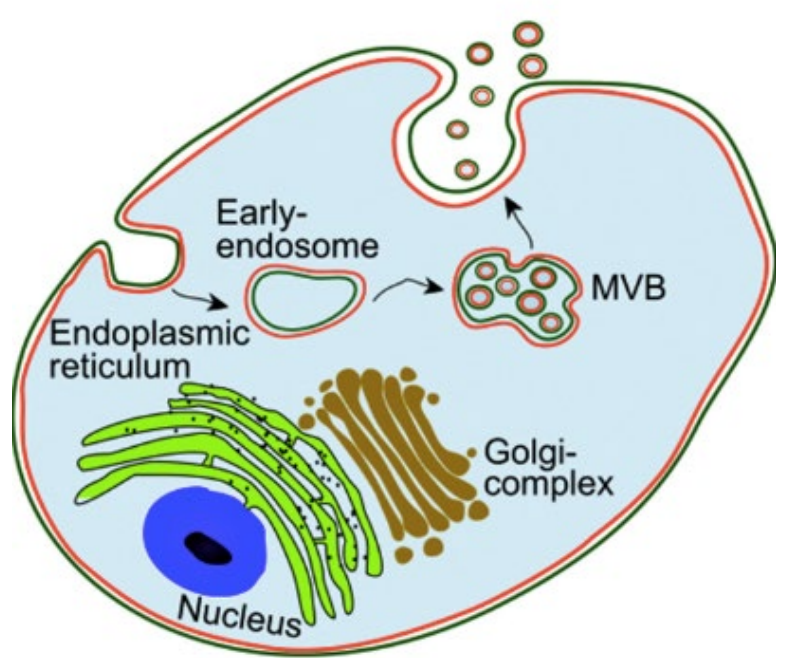

Figure 1-2. Formation and release of exosomes from cells. Figure (Skotland, Sandvig, \& Llorente, 2017) reprinted from the PhD degree of Santosh Phuyal, University of Oslo.

Exosomes are membrane vesicles enclosed in a lipid bilayer. These nanoparticles are released by most mammalian cell for intercellular communication, and are unique to the cell in which they originate (De la Torre Gomez et al., 2018). EVs circulate in human biological fluids such as blood, urine, saliva, and human milk (De la Torre Gomez et al., 2018). There are three steps in exosome biogenesis: biogenesis of MVBs, the transport of MVBs to the plasma membrane, and the fusion of the MVBs with the plasma membrane (Figure 1-2), which releases the intraluminal vesicles from the MVBs. Exosomes are the smallest of the three types of EVs, the other two being microvesicles and apoptotic bodies (T Skotland et al., 2017).

The function of EVs was further emphasized due to their touted role in cancer progression. In lung cancer, platelet derived microvesicles transferred an integrin (CD41) to numerous lung cancer lines, eliciting the highest response in highly metastatic cells, which indicated the significance of extracellular vesicles in disease pathology (Janowska-Wieczorek et al., 2005). EVs contain microRNA and messengerRNA. When mouse EV-derived RNA was transferred to human mast cells, new mouse proteins were found in the recipient cells, indicating 
that the transferred mRNA was able to be translated after entering a new cell (Valadi et al., 2007). Bovine milk derived EVs were used for drug delivery because EVs from bovine milk can be obtained more cost-effectively and on a larger scale (Munagala, Aquil, Jeyabalan, \& Gupta, 2016). Bovine milk EVs were also found to have cross-species tolerance; when mice possessing human lung cancer (A549) xenografts were treated with the cancer drug Withaferin A (WFA) as free-WFA or exo-WFA (drug-loaded EVs), the exo-WFA exhibited a significantly greater tumor inhibitory effect (46\% vs $23 \%$ ) than the free WFA (Munagala et al., 2016).

Given the public health importance of cancer and drug research, the study of EVs in other fields has been neglected. For example, (T Skotland et al., 2017) analyzed approximately 280 lipid species in the metastatic prostate cancer cell line PC-3 (Llorente et al., 2013) and stated that to their knowledge, the only study providing quantitative data for both the parent cell and exosomal lipid composition of more than just a few lipid species was their own, which still holds true. There is therefore a general gap in knowledge regarding the application of EVs to other fields such as nutrition. There is also limited knowledge on fatty acid constituents of extracellular vesicles, which may be involved in cellular processes such as EV release and formation.

\section{Lipids and fatty acids in extracellular vesicles}

Lipids in the plasma membrane may influence the formation and release of the EVs during the biogenesis of EVs. Compared to the cell from which it is derived, lipids such as cholesterol and sphingomyelin are enriched in EVs (Llorente et al., 2013). The intraluminal vesicles of multivesicular endosomes are sorted into lysosomes for degradation of cargo, or secreted as EVs (Trajkovic et al., 2008), a process that is dependent on the composition of lipids. It was found that the transfer of exosome-associated domains into the lumen of the endosome 
required the sphingolipid ceramide, which implicated lipids in the formation and subsequent communication functions of EVs (Trajkovic et al., 2008). EV synthesis, release and transport occur in a highly regulated manner and thus it is plausible that alteration of lipids composition of EV may alter function.

A study quantifying over 217 lipids in the EVs of PC-3 cells showed differences in the relative proportion of lipids of EVs compared to the cells of origin (Llorente et al., 2013). The total lipid concentration in protein was $94.2 \pm 6.8 \mathrm{in} \mathrm{PC}-3$ cells and $790.7 \pm 68.5 \mathrm{in} \mathrm{nmol} / \mathrm{mg}$ in EVs, indicating a greater-than 8-fold enrichment of lipids into EVs per mg protein (Llorente et al., 2013). Additionally, it was discovered that the relative proportions of glycosphingolipids, cholesterol, sphingomyelin (SM), and phosphatidylserine (PS), was over 15 times higher in the EVs than in the cell of origin (Llorente et al., 2013). The membrane of EVs is highly ordered, and the high composition of glycosphingolipids in EVs likely confers EVs with stability in extracellular environments, which is essential for their role in cell-to-cell communication. Lipids in EVs have also been associated with biogenesis and communication due to the presence of lipid raft associated proteins such as flotillin-1; lipid rafts are areas of the plasma membrane rich in cholesterol and glycosphingolipids (de Gassart, Geminard, Fevrier, Raposo, \& Vidal, 2003). The tetraspanins CD9 and CD81 have also been found to be physically and functionally linked with cholesterol; tetraspanins are believed to form a tetraspanin "web" of molecular interaction. Tetraspanin/tetraspanin complexes were precipitated using digitonin, a cholesterol precipitating reagent (Charrin et al., 2003). Due to the interaction of tetraspanin with cholesterol, it was postulated that tetraspanins in EVs may be attributed to the association with lipid rafts (Brzozowski et al., 2018). 
Inconsistency in methods of EV isolation and analysis have hindered the acquisition of knowledge pertinent to the significance of the lipid composition of EVs (T Skotland et al., 2017). Results from the different techniques are dependent on not just the method used, but also the purity of the samples being analyzed (T Skotland et al., 2017). Additionally, lipids present in EVs isolated from different biofluids vary, including EVs from the same biofluid in healthy controls and diseased patients (Table 1-1). Consistent isolation and characterization methods for EVs and constituents of EVs from different biofluids, including complex matrices such as human milk, are therefore necessary to understand the specific function of lipid compositions that have been observed in lipid bilayers of EVs. Although the present study does not explore the composition of fatty acid-enriched fractions of EVs, the validation of an isolation method for EV isolation in human milk will facilitate such future studies on the structure and functional significance of EVs in human milk. 
Table 1-1. Summary of lipids and fatty acids in EVs in the literature.

\begin{tabular}{|c|c|c|c|c|}
\hline $\begin{array}{l}\text { Origin of } \\
\text { EVs }\end{array}$ & $\begin{array}{l}\text { Type of Lipid(s) } \\
\text { Studied }\end{array}$ & Focus of Study & Key Findings and Conclusions & Citations \\
\hline Urine & $\begin{array}{l}107 \text { lipid species } \\
\text { were quantified } \\
\text { using mass } \\
\text { spectrometry } \\
\text { quantitative } \\
\text { lipidomic analysis. } \\
\text { Lipid species refer } \\
\text { to lipid molecules } \\
\text { with different fatty } \\
\text { acyl groups. } \\
\text { Phosphatidylserine } \\
\text { and } \\
\text { lactosylceramide } \\
\text { were significantly } \\
\text { higher in cancer } \\
\text { patients compared } \\
\text { to healthy } \\
\text { controls. }\end{array}$ & $\begin{array}{l}\text { Exosomes are non- } \\
\text { invasive biomarkers } \\
\text { for cancer. } \\
\text { The use of lipids in } \\
\text { urinary exosomes of } \\
\text { prostate cancer } \\
\text { patients was } \\
\text { investigated. }\end{array}$ & $\begin{array}{l}\text { Of the } 107 \text { lipid species, } 36 \text { of the } \\
\text { most common lipid species were } \\
\text { further analyzed, } 9 \text { of which } \\
\text { significantly differed between } \\
\text { cancer patients and healthy controls. } \\
\text { Lipids in urinary exosomes may be } \\
\text { useful as biomarkers for prostate } \\
\text { cancer since the high patient: } \\
\text { control ratio of specific lipids such } \\
\text { as phosphatidylserine distinguished } \\
\text { the cancer patients from controls. }\end{array}$ & $\begin{array}{l}\text { (T. Skotland } \\
\text { et al., 2017) }\end{array}$ \\
\hline $\begin{array}{l}\text { Exosomes } \\
\text { secreted by a } \\
\text { parasite } \\
\text { internalized }\end{array}$ & $\begin{array}{l}\text { Plasmalogens, } \\
\text { other } \\
\text { glycerophospholip } \\
\text { ids, cholesterol, }\end{array}$ & $\begin{array}{l}\text { Since lipids are key } \\
\text { constituents of } \\
\text { exosomes given that } \\
\text { the lipid bilayer of }\end{array}$ & $\begin{array}{l}\text { Plasmalogens and other classes of } \\
\text { glycerophospholipids were enriched } \\
9 \text { to } 62 \text { fold, and a comparative lack } \\
\text { of cholesterol and SM in the }\end{array}$ & $\begin{array}{l}\text { (Simbari, } \\
\text { 2016) }\end{array}$ \\
\hline
\end{tabular}


by mouse epithelial cells

Skeletal
muscle
derived
microvesicles
(SkMV)
collected
from plasma

and sphingomyelin (SM).

Long-chain fatty
acids (LCFA)
acids (LCFA)

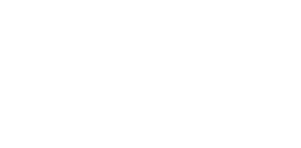

exosomes is exposed to the environment, the composition of parasite-secreted EVs were compared to exosomes secreted by a murine host. Ultraperformance liquid chromatography $\square$ tandem mass spectrometry (UPLC$\mathrm{MS} / \mathrm{MS}$ ) was used for lipid analysis.

Investigate whether or not acute exercise (a single bout) influences SkMV expressing LCFA transport proteins CD36 (SR-B2) and FATP4 in obese males with type II diabetes and obese male controls. parasitic exosomes was observed compared to the murine exosomes. Analyses of exosomal membrane dynamics indicated the membrane of the nematode was more rigid.

The parasite may maintain rigidity of the exosomal membrane from increased plasmalogens compared to other lipids, plasmalogens are highly prevalent in some EVs.

Nielsen et al. (2019) found a

positive correlation between

(Nielsen et SKMVs carrying CD36 and performance of acute exercise only in males with type II diabetes. The authors conclude that studying LCFA transport protein released by EVs in "real-time" could be key to understanding LCFA uptake in subjects that have impaired glucose metabolism. al., 2019) 


\section{Isolation of EVs, quantification, and verification}

A variety of methods can be used to isolate exosomes. However, there are trade-offs associated with each method. Table 1-2 summarizes the applications of common EV isolation methods, indicating advantages and disadvantages of methods for different applications. A commercial kit makes sense technically for applications where there is a high sample number, and low volume for each sample.

Table 1-2. Summary of the applications of different exosome isolation techniques.

\begin{tabular}{|c|c|c|c|c|}
\hline Method & Principle & $\begin{array}{l}\text { Best Used } \\
\text { When }\end{array}$ & Challenges & Citations \\
\hline $\begin{array}{l}\text { Ultracentrifugation } \\
\text { (UC) }\end{array}$ & $\begin{array}{l}\text {-Centrifuge at } \\
\text { increasing } \\
\text { speeds, } \\
\text { eliminating } \\
\text { large cells and } \\
\text { cell debris; } \\
\text { final pellet is } \\
\text { exosomes }\end{array}$ & $\begin{array}{l}\text {-Larger } \\
\text { volumes (mL) } \\
\text { of biofluid are } \\
\text { available } \\
\text {-Optimal speed } \\
\text { for purity and } \\
\text { yield of } \\
\text { specific } \\
\text { biofluid can be } \\
\text { tested or is } \\
\text { known }\end{array}$ & $\begin{array}{l}\text {-May not be } \\
\text { capable of } \\
\text { differentiating } \\
\text { exosomes from } \\
\text { contaminants } \\
\text { (larger vesicles, } \\
\text { protein and lipid } \\
\text { aggregates). } \\
\text {-Time consuming } \\
\text {-Difficult to } \\
\text { isolate exosomes } \\
\text { from large } \\
\text { numbers of } \\
\text { samples due to } \\
\text { space constraints }\end{array}$ & $\begin{array}{l}\text { (SBI, 2018a; T } \\
\text { Skotland et al., 2017; } \\
\text { Thery, Clayton, } \\
\text { Amigorena, \& } \\
\text { Raposo, 2006) }\end{array}$ \\
\hline Commercial Kits & $\begin{array}{l}\text {-Proprietary } \\
\text { polymer that } \\
\text { precipitates } \\
\text { exosomes and } \\
\text { microvesicles } \\
\text { between } 30 \text { and } \\
200 \mathrm{~nm}\end{array}$ & $\begin{array}{l}\text {-When smaller } \\
\text { amounts of } \\
\text { biofluid are } \\
\text { available } \\
\text {-Specialized } \\
\text { equipment } \\
\text { (UC) is not } \\
\text { available and } \\
\text { there are many } \\
\text { samples from }\end{array}$ & $\begin{array}{l}\text {-Multiple } \\
\text { commercial kit } \\
\text { options } \\
\text { available } \\
\text {-No commercial } \\
\text { kits are } \\
\text { specifically } \\
\text { optimized for } \\
\text { human milk }\end{array}$ & (SBI, 2018a, 2018c) \\
\hline
\end{tabular}


which EVs will

be isolated

\begin{tabular}{lllll} 
Immunomagnetic & -Magnetic & -Highly pure & -If different & \\
beads are & exosomes are & exosome & \\
coated with & needed for & markers are less & (Pedersen et al., \\
antibodies & downstream & prevalent in a & 2015) \\
& against & application & particular & \\
common & & biofluid, beads & \\
exosomal & & may isolate & \\
markers (CD9 & & fewer \\
& & & exosomes . & \\
\hline
\end{tabular}

The optimal method of EV isolation may vary depending on the specimen from which the EVs are isolated. It is therefore important to consider the methods utilized for EV isolation when interpreting the results of a study. To isolate EVs using differential UC, initial steps eliminate large dead cells and cell debris by centrifuging at increasing speeds, discarding the pellet and keeping the supernatant. The final pellet, which appears after centrifuging at 100,000 $\mathrm{x}$ $\mathrm{g}$, contains the exosomes, and is washed in phosphate buffered saline (PBS) and centrifuged one more time to eliminate contaminating proteins (Thery et al., 2006). Although UC is the most commonly used EV isolation method (Sáenz-Cuesta et al., 2015; Xin Wang, 2017; Yamada, Inoshima, Matsuda, \& Ishiguro, 2012), it can result in the co-isolation of exosomes, lipoparticles, and lipid droplets, which alters the lipid composition of EVs as opposed to isolating exosomes with a typical membrane bilayer (T Skotland et al., 2017). Therefore, if the downstream application of exosome isolation involves lipid analysis, another method of isolation, such as a commercial kit should be used instead.

UC has been considered the gold-standard for exosome isolation due to its perceived ease of use and affordability (Li, Kaslan, Lee, Yao, \& Gao, 2017). However, UC is time-consuming, requires large volumes of biofluid and specialized equipment, and EVs isolated by UC may also 
be contaminated by proteins (Caradec et al., 2014). The isolation of EVs using UC was compared, and it was discovered that centrifuging at different speeds impacts the purity and yield of the EVs that are isolated, since undesirable contaminants such as protein aggregates may sediment similarly to EVs (Jeppesen et al., 2014). It was found that the optimal speed for purity and yield differed between two cell lines. Therefore, when a standard UC protocol is utilized to isolate EVs from a variety of biofluids, it may not be optimized to collect EVs with the greatest purity and yield from human milk.

Other methods of EV isolation include precipitation-based methods and immunoprecipitation. Combination methods, such as UC followed by use of a commercial kit can also be performed. One precipitation method works by using a proprietary polymer reagent that precipitates EVs between 30 and $200 \mathrm{~nm}$. According to the manufacturer, the kit eliminates the trade-offs of yield, purity, and speed, and it can be used with as little as $250 \mu \mathrm{L}$ of specific biofluids (SBI, 2018c). However, the commercial kits available have not been authenticated for use of human milk; ExoQuick ${ }^{\circledR}$ is recommended for plasma, serum, and ascites fluid, while ExoQuick $^{\circledR}$ TC is recommended for isolating EVs from tissue culture (SBI, 2018c). 


\section{a. $\mathbf{U C}$}

Sample collection

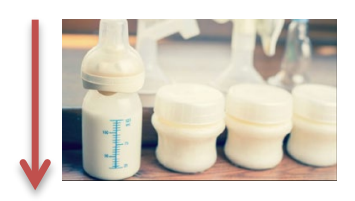

Defat milk (centrifuge $2000 \mathrm{x}$ g for $10 \mathrm{~min}$ )

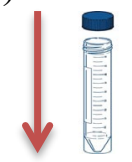

Centrifuge $10,000 \mathrm{x} \mathrm{g}$ for $30 \mathrm{~min}$

Keep supernatant: pellet $=$ cell

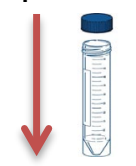

Ultracentrifuge $100,000 \mathrm{x}$ g for $70 \mathrm{~min}$ Pellet $=$ exosomes + contaminating proteins

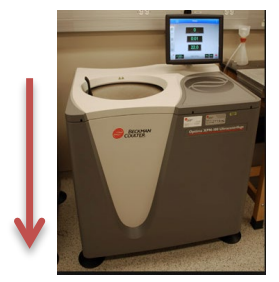

Wash in $1 \mathrm{X}$ PBS and ultracentrifuge $100,000 \mathrm{xg}$ for $70 \mathrm{~min}$
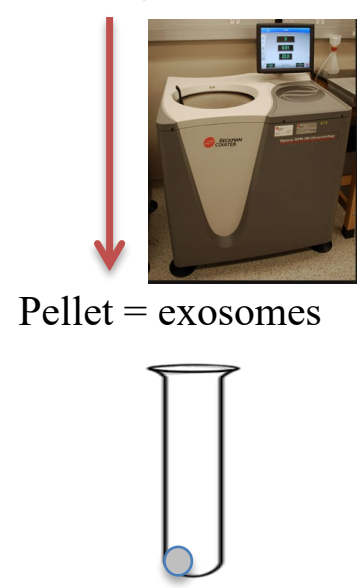

\section{b. Commercial Kit}

Sample collection

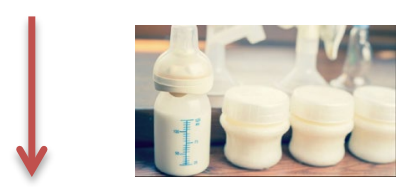

Defat milk

(centrifuge $2000 \mathrm{x} \mathrm{g}$ for $10 \mathrm{~min}$ )

then $3,000 \mathrm{x} g$ for $15 \mathrm{~min}$

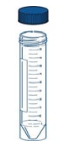

Centrifuge supernatant at 12,000

$\mathrm{x} \mathrm{g}$ for $30 \mathrm{~min}$ and discard

debris; filter

Add ExoQuick TC TM and incubate overnight at $4{ }^{\circ} \mathrm{C}$

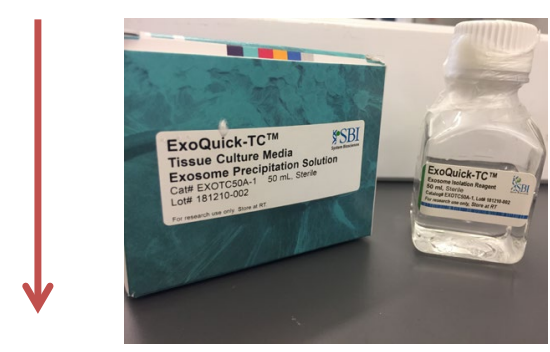

Centrifuge at 5,000 $\mathrm{x}$ g for $30 \mathrm{~min}$, and re-suspend in

If desired, purify further with ExoQuick TC TM

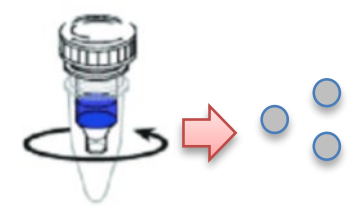

Figure 1-3. Comparison of UC with a commercial kit for isolation of EVs in human milk. UC adapted from (Thery et al., 2006) and ExoQuick TC TM(Xin Wang, 2017). 
When isolating EVs, the downstream application and need for intact structure and high yield must be considered. Using ExoQuick ${ }^{\circledR}$ precipitation, UC with ExoQuick ${ }^{\circledR}$ precipitation, and UC with density gradient centrifugation, and a protocol for isolating EVs in human milk (Admyre et al., 2007) with bovine milk, it was found that the human milk isolation protocol failed to isolate EVs from the bovine milk using the ExoQuick ${ }^{\circledR}$, as they were unable to suspend the final EV pellet in PBS for storage due to interference of precipitating proteins (Yamada et al., 2012). The method was unsuccessful, because the de-fatted bovine milk could not be filtered, perhaps due to the greater protein content of bovine milk, which clogged the filter. Therefore subsequent isolation steps could not be performed. UC with ExoQuick ${ }^{\circledR}$ and precipitation resulted in the highest yield, while density gradient centrifugation resulted in higher quality EV isolation, with more intact morphological structures, as visualized by electron microscopy. Given the advantages and disadvantages of each method, the downstream application should be considered when choosing a method. For example, to analyze total fatty acid content in human milk, yield is more important than obtaining intact vesicles.

The increased recovery associated with UC and ExoQuick ${ }^{\mathrm{TM}}$ precipitation relative to EVs isolated with $\mathrm{UC}$ and density gradient centrifugation may be useful to analyze mRNA kinetics or exosomal proteins (Yamada et al. 2012). Conversely, the higher purity isolations from UC with density gradient centrifugation relative to UC and ExoQuick ${ }^{\mathrm{TM}}$ precipitation may be more appropriate for analysis of exosomal proteins. The Yamada et al. (2012) study highlights the importance of selecting an EV isolation technique based on careful consideration of the type of specimen from which EVs are being isolated, as well as the downstream application and targeted use. 


\section{EV verification and quantification methods}

After isolating EVs, methods used for verification include immunoblot and electron microscopy. Immunoblot has been used to confirm EV isolation by detecting the presence of EV markers such as CD133 and CD63 (J. Kim, Tan, \& Lubman, 2015; Sáenz-Cuesta et al., 2015; Yamada et al., 2012) and the absence of cellular contamination (SBI, 2018a). Using electron microscopy, EV isolation can be confirmed by observing the characteristic size and shape of the vesicles (Lobb et al., 2015; Samuel et al., 2017; Sáenz-Cuesta et al., 2015).

Nanoparticle Tracking Analysis (NTA) is used to measure particle size and concentration and confirm the isolation of EVs (Hock et al., 2017). EV quantification is also performed using the Bicinchoninic Acid (BCA) protein quantification assay (Thermo Scientific, IL, USA). This method makes the assumption that protein concentration correlates positively with the concentration of EVs. The amount of EV protein positively correlates to a greater volume of biofluid (J. Kim et al., 2015), which supports this assumption. However, this assumption is a limitation because the relative abundance of EVs between different types of specimen or between isolation methods cannot be inferred.

Measuring protein concentration in EVs is not always an accurate method of EV quantification although it has been frequently used. EVs isolated with UC were shown to have a high albumin content compared to EVs isolated with a precipitation method, impairing the utilization of protein quantification as a way to accurately quantify EVs (Caradec et al., 2014). EV protein content quantified with BCA correlated with serum volume in EVs isolated with UC and a precipitation method (Caradec et al., 2014). However, when NTA was used to quantify nanoparticles, there was no correlation between the particle number isolated by UC and the original volume of serum. This suggests that albumin concentration confounded the protein concentration 
measurements. Only comparing the protein contents of EVs isolated with the same technique can help eliminate the issue of misleading protein quantification results Additionally, EV confirmation and quantification methods may be more suited to a particular biofluid; for example, it was found that detection of CD133 markers for Western blot was better with EVs from urine than from blood (Sáenz-Cuesta et al., 2015).

\section{Functions of milk EVS}

EV isolation techniques for human milk need to account for components of the milk matrix, such as proteins, which could influence the success of an isolation technique. Additionally, if comparing EVs isolated from milk separately, the timing of obtaining the milk post-partum should also be considered. Human milk EVs from early and mature human milk were compared, investigating the influence of lifestyle and allergic sensitization on the profile of the EVs (Torregrosa Paredes et al., 2014). There were higher levels of HLA-DR antibodies and a lower concentration of EV protein and a lower number of vesicles in early milk (days 3-8 postpartum) than in mature milk (two months postpartum). These differences in EV composition and quantity throughout lactation should be considered according to the downstream analysis.

It was also found that there were over 4980 more proteins implicated in immune response and growth in EVs from bovine colostrum that were not present in mature milk. They were significantly enriched $(p<0.05)$ with Rab proteins known for their role in vesicle docking and fusion as well as with proteins associated with the innate immune response (Samuel et al., 2017). Further research on these findings may be important to determine if the same differences in proteins occur in human colostrum and mature milk. Commercially, this knowledge is an important consideration so manufacturers of infant formula can make consistent products. Likewise, it was concluded that processing of milk by dairies significantly results in loss of 
miRNAs; pasteurization and homogenization of whole milk decreased miR-200c concentration $63 \pm 28 \%$ (Howard et al., 2015). Loss of miRNA during homogenization was attributed to lysis or disruption of EV membranes caused by the shear force applied during homogenization (Howard et al., 2015). MiRNA loss is problematic, as EVs are involved in cell-to-cell communication with their cargo, which can include miRNA (Munagala et al., 2016).

In addition to regulation of immune response and inflammation, EVs in milk promote epithelial growth in the intestine, perhaps because the phospholipid membrane allows the contents of EVs to reach the intestinal lumen of neonates (Hock et al., 2017a). Necrotizing enterocolitis (NEC) is the most common gastrointestinal emergency in newborns, and consumption of human milk has been associated with decreased risk of NEC. The impact of milk derived EVs on intestinal epithelial cell viability was therefore investigated. Utilizing Exoquick $^{\mathrm{TM}}$ reagent, EVs were isolated from rat milk and a rat small intestine epithelial cell line (IEC-18) was treated with $0.5-\mu \mathrm{g} / \mu \mathrm{l} \mathrm{EVs,} \mathrm{EV-free} \mathrm{milk,} \mathrm{or} \mathrm{an} \mathrm{EV-free} \mathrm{control} \mathrm{solution,} \mathrm{finding}$ that the cells treated with EVs significantly increased IEC viability measured using a colorimetric assay compared to EV free milk and the control ( $p<0.05$ ) (Hock et al., 2017a).

\section{Challenges in the study of human milk EVS}

Human milk is frequently refrigerated at $4{ }^{\circ} \mathrm{C}$ or frozen at -20 for feeding infants, and at $-80{ }^{\circ} \mathrm{C}$ for research purposes and milk banking. Studying human milk samples that were processed immediately after they were obtained and samples stored for two hours at $4{ }^{\circ} \mathrm{C},-20$ and $-80{ }^{\circ} \mathrm{C}$ temperatures, it was discovered that the number of viable cells collected immediately was donor dependent (Zonneveld et al., 2014). It was found that cell death and subsequent formation of cell debris impacted the total pool of milk EVs. This could be problematic because 
contamination of the native milk EV with storage-induced EVs can occur if storage induced stress or cell death induces the formation of new EVs. The disintegration of dead cells can result in the formation of membrane-enclosed vesicles (Zonneveld et al., 2014). Human milk was spiked with cells of murine origin to track the formation of storage induced EVs. EVs were either isolated from the milk right away or stored at $-80{ }^{\circ} \mathrm{C}$. A ten to one hundred fold increase in the murine CD9 and up to a 2,000\% increase in murine MHC Class II EV markers stored at $80{ }^{\circ} \mathrm{C}$ was observed compared to the immediately processed samples. This suggests the presence of a newly formed pool of vesicles released by cells during storage that contaminated the pool of EV naturally occurring in milk (Zonneveld et al., 2014).

Although the Zonneveld et al. (2014) study raises important considerations for researchers, it is not feasible to eliminate these concerns when researching human milk, as the only way to do so would be to isolate and study EVs immediately after a mother produced milk. Some commercial EV isolation kits require an overnight incubation step at $4{ }^{\circ} \mathrm{C}$, and it was found that the viable cells were decreased after only two hours (Zonneveld et al., 2014). Because it is not feasible to complete all the necessary analyses and avoid storage of human milk, consistency is crucial, meaning any aliquots of human milk that are compared should have undergone identical freeze-thaw processes.

In summary, EVs in human milk may have important functions like mediation of inflammatory, immune, and epithelial responses. Since lipids and lipid metabolizing enzymes take part in EV release and formation, fatty acid content may dictate the function of EVs. However, in order to even carry out structure and function research, a consistent EV isolation technique suited for the specimen being studied and downstream application is needed. Although use of a commercial isolation kit to isolate EVs from human milk is a viable option, this method 
has not yet been validated for human milk. Therefore, before structure and function of EVs can be explored to benefit manufacturers of commercial infant formula products and breastfeeding mothers, it is crucial that this isolation method is validated for future use. 


\section{Rationale and significance}

Although human milk is the recommended source of nutrition for most infants, there are a variety of reasons a parent may need to feed with commercial infant formula to supplement or replace human milk. Thus, understanding if and how bioactive components of human milk provide optimal nutrition for infants represents a public health interest that is particularly relevant to parents and commercial manufacturers of infant formula seeking to replicate the unique benefits of human milk in products. The present study will address this public health issue by building upon the established knowledge that fatty acid deficiency has been linked to deleterious health outcomes, and that the development of a suitable isolation technique for EVs in human milk has hindered advances in research regarding structural and functional applications.

It was hypothesized that bioactive components in human milk are related to health outcomes. The hypothesis was tested through the following objectives:

(1): Determine whether fatty acid composition of milk relates to infant developmental outcomes reported by the BSID-III.

(2): Develop a feasible method of EV isolation from human milk that satisfies the International Society of Extracellular Vesicles (ISEV) criteria.

This project has widespread clinical and public health implications, as it could impact maternal nutrition guidelines in the perinatal period. The proposed research will also contribute to the product development sector. It is widely accepted that commercial infant formula is inferior to human milk with respect to several health outcomes. Therefore, if fatty acids in human milk are correlated to superior developmental outcomes corroborated by standard tests, 
the results of the proposed study can help product developers ameliorate the nutritional quality of their infant formula. Furthermore, this project will improve knowledge on EV isolation in human milk, which has not been studied as extensively as EV isolation in other biofluids such as blood and urine. If the results of the proposed study indicate that the essential fatty acid composition of EVs in human milk and the fatty acid composition of human milk are associated with favorable infant health outcomes recorded by standardized health tests, this could pave the way for maternal dietary recommendations that would favorably manipulate the fatty acid composition of human milk and EVs in milk. 


\section{The relation between the fatty acid composition of human milk and BSID-III developmental outcomes}

\section{Introduction}

Human milk is the recommended source of infant nutrition for the first six months of life (CDC, 2018). The consumption of human milk compared to infant formula has been correlated with numerous short and long-term benefits, including immune system development, reduced risk of gastrointestinal and respiratory diseases, superior cognitive development, and reduced risk of chronic diseases such as diabetes, obesity, and hypertension (Vergilio Visentainer et al., 2018). Specific benefits of human milk, such as cognitive benefits, have been linked to long chain fatty acids such as DHA and ARA, which make up about $20 \%$ of the brain's fatty acid content and are involved in neurodevelopment (Pang et al., 2020). The omega-3 and omega-6 PUFAs are important for human health, growth, and development. Infants consuming colostrum higher in linoleic acid and lower in DHA exhibited lower IQ scores at five to six years of age (Bernard et al., 2017). Omega-3 PUFAs are derived from alpha linolenic acid (ALA, 18:3n-3), while omega-6 PUFAs are derived from linoleic acid (LA, 18:2n-6). Because the precursor molecules ALA and LA cannot be endogenously synthesized by humans, they are considered essential. Longer chain fatty acids are synthesized very slowly from the precursor molecules. Less than $0.1 \%$ of LA is converted into ARA (Heaton, Meldrum, Foster, Prescott, \& Simmer, 2013). For this reason, consuming pre-converted long chain PUFAs through diet can be a more efficient way to obtain health benefits.

The Bayley Scales of Infant and Toddler Development - Third Edition (BSID-III) is a developmental assessment that can be administered to children between one and 42 months of 
age. It is comprised of the Cognitive, Language, Motor, Social-Emotional, and Adaptive Behavior Scales. Based on the composite scores in the Cognitive, Language, and Motor Scales, the assessment identifies signs of developmental delay (Bayley, 2006). The Language Scale assesses receptive and expressive language, and the Motor Scale examines both Fine and Gross Motor skills (Bayley, 2006).

Previous work has associated higher Mental Development Index scores, a BSID-III predecessor to the Cognitive Composite, with longer-term consumption of human milk (Angelsen et al., 2001). Is has also been found that infants whose mothers supplemented with DHA while breastfeeding exhibited a higher Bayley-II Psychomotor Index (predecessor to Bayley-III Motor Composite) at 30 months of age (Jensen et al., 2005). Findings from these studies indicate the association between the consumption of human milk and cognitive and motor benefits during infancy and childhood. These associations also highlight the need for research to discover which components in human milk are responsible for these benefits.

Although human milk has been associated with a multitude of acute and long-term benefits, many of the correlations between human milk and superior cognitive functioning may be confounded by other factors associated with breastfeeding, such as maternal socioeconomic status and IQ (Walfisch, Sermer, Cressman, \& Koren, 2013) The practice of consuming milk directly from the breast versus pumped milk may also moderate these benefits. Due to the gap in knowledge that has resulted from challenges in objectively studying human milk, there is a need to relate the fatty acid constituents of human milk to infant health and developmental outcomes using a consistent evaluation such as the BSID-III. It is also necessary to consider proportions of fatty acids within the milk matrix, as the benefits conferred by single fatty acids may depend on the presence or absence of other fatty acids. Infant movement quality, for example, which can be 
used to assess neurological development in young infants, was found to be sensitive to the maternal dietary balance of DHA and ARA. When lactating mothers supplemented with just DHA, $61 \%$ of the infants exhibited mildly abnormal general movements. Only $31 \%$ of infants in the control group (meaning the mother was not supplementing with DHA or ARA) and 34\% of infants whose mothers were supplementing with both DHA and ARA exhibited mildly abnormal general movements. (van Goor et al., 2010). In the United States, infant formula generally contains ARA to DHA in a 2:1 ratio, since human milk typically contains greater concentration of ARA than DHA (Lien et al., 2018). However, more research is needed to determine if the proportions of these and other fatty acids are optimal for infant health and development.

The objective of the present study was to determine whether the relative amounts of unsaturated fatty acids in human milk correlated with the Cognitive, Motor, and Language Composite outcomes reported by the BSID-III. It was hypothesized that there would be a correlation between greater concentrations of omega-3 EFAs in milk and positive developmental outcomes reported by the BSID-III. Elucidating the importance of specific fatty acids and fatty acid ratios in human milk represents a public health interest. Knowledge of the optimal composition of human milk may facilitate dietary recommendations for breastfeeding mothers and aid in the manufacture of more nutritious infant formula products.

\section{Methods}

\section{Acquisition of human milk and developmental data}

The studies involving human participants were reviewed and approved by Chapman University IRB. The patients/participants provided their written informed consent to participate in this study (Chapman University REB approval number 1314H044). 
Mothers were recruited by asking eligible women receiving care at University of California, Irvine clinics in Orange County, California (USA). Inclusion criteria for participating in the study included: English-speaking, absence of alcohol or drug use during pregnancy, absence of endocrine, hepatic, or renal disorders, over 18 years of age, and a singleton pregnancy. Women with a disorder related to uterine or cervical function were excluded. Milk samples were obtained between 2015 and 2017, and provided between $~ 9: 00$ a.m. and 4:30 p.m. The samples were stored at $-80^{\circ} \mathrm{C}$ upon collection. The data collected included the Cognitive, Language, and Motor Scales of the BSID-III. Cognitive, Language, and Motor Composite scores were obtained at $\operatorname{six}(n=60,61$, and 61$)$ and 12 months of age $(n=61,61$, and 61$)$ respectively. Data on the proportion of human milk feeding was obtained by interview. Data specifying socioeconomic status, sex of the infant, maternal age and education level, parity, ethnicity, and cohabitation with the baby's father was also obtained by interview.

\section{Fatty acid analysis of human milk}

Fatty acid analysis of human milk aliquots $(\mathrm{n}=70)$ was performed at University of California, San Diego Lipidomics Core (San Diego, CA, USA). Fatty acids were extracted from $0.5 \mu \mathrm{L}$ human milk samples with butanol-methanol extraction (BUME). After solvent removal, samples were saponified by adding $250 \mu \mathrm{L} \mathrm{MeOH}$ and $250 \mu \mathrm{L} 1 \mathrm{~N} \mathrm{KOH}$, incubating for 60 min at $37^{\circ} \mathrm{C}$, and neutralizing the $\mathrm{pH}$. Internal standards were added, and fatty acids were extracted with isooctane. Upon removal of the solvent, fatty acids were derivatized by adding $25 \mu \mathrm{L} 1 \%$ PFBB and $25 \mu \mathrm{L} 1 \%$ DIPEA. Samples were incubated at room temperature for $20 \mathrm{~min}$, solvent was removed, $50 \mu \mathrm{L}$ iso-octane was added, and samples were transferred to MS vial with insert. Total esterified and non-esterified fatty acids were quantified using GC-MS (Agilent 5975 GC/Mass Spectrometer, Mass Hunter, Multiquant software) using an injection volume of $1 \mu \mathrm{L}$. 
The present study reports on 22:6n-3 (DHA), 20:5n-3 (EPA), 20:4n-6 (ARA), 18:3n-3 (ALA), 18:2n-6 (LA), and 18:1n-9 (OA). The absolute fatty acid concentrations were converted to relative percentages for subsequent analysis.

\section{Statistical Analysis}

Statistical analysis was performed using IBM SPSS ${ }^{\circledR}$ Version 25. Descriptive statistics were calculated for sample population characteristics, BSID-III outcomes, and relative concentrations of fatty acids in milk. A simple linear regression analysis was used initially to model the relation between the relative percentage of the selected fatty acids and the Bayley Cognitive, Motor, and Language Composite scores at six and 12 months of age before incorporating covariates and modifiers into the regression model. Maternal age, self-reported years of education, ethnicity (Latina, non-Latina), infant sex, income to needs ratio (INR) and cohabitation with the baby's father were considered as covariates by correlating with predictor and response variables. Maternal age, education, ethnicity, and INR covariates were chosen to be incorporated into the regression model.

The percentage of infant diet comprised of human milk at six months was analyzed as a modifier between the relative proportions of fatty acid and the Bayley outcomes. Modifier values were incorporated into the regression model, and the p-value of the interaction term between modifier and predictor was assessed to determine if proportion of milk consumption in diet modified the relation between fatty acids and BSID-III outcomes. For each regression model incorporating the modifier, all interaction terms were created with mean-centered variables. A significance level of $\alpha=0.05$ was used throughout the study. The following sample size calculation was performed to detect a $16 \%$ (clinically significant) difference in any Bayley 
subscale score at $80 \%$ power in one-tail testing at a $5 \%$ level of significance using previously reported mean and standard deviation (Badr, 2009).

$\mathrm{n} \approx\left[\left(2 *\left(\mathrm{Z}_{\text {power }}+\mathrm{z}_{1-\mathrm{a}}\right)\right) \div\left(\left(\mu_{1}-\mu_{2}\right) / \delta\right)\right]^{2}$

$\mathrm{n} \approx[(2 *(0.8416+1.96)) \div(16 / 15)]^{2}$

$\mathrm{n} \approx 35$ (per timepoint)

Equation 3-1. Sample size calculation for determination of clinically significant differences in Bayley subscale score means. 


\section{Results}

Table 3-1. Maternal and demographic information and categorical level of education for the 70 mother-child pairs in the present study. INR is reported as percent above or below the poverty line.

\begin{tabular}{|c|c|c|c|}
\hline $\begin{array}{l}\text { Infant and Maternal } \\
\text { Demographic }\end{array}$ & Values & $\begin{array}{l}\text { Highest Level } \\
\text { of Maternal } \\
\text { Education } \\
\text { Attained } \\
(\mathbf{n}=70)\end{array}$ & $\begin{array}{l}\text { Number of } \\
\text { Participants } \\
\text { (\%) }\end{array}$ \\
\hline Infant characteristics & & $\begin{array}{l}\text { Primary, } \\
\text { elementary, or } \\
\text { middle school }\end{array}$ & $3(4.3 \%)$ \\
\hline Male, $n$ & 35 & $\begin{array}{l}\text { High school or } \\
\text { GED } \\
\text { equivalent }\end{array}$ & $11(15.7 \%)$ \\
\hline Female, $n$ & 35 & $\begin{array}{l}\text { Technical or } \\
\text { vocational } \\
\text { school }\end{array}$ & $7(10 \%)$ \\
\hline Birth order (\%) & & $\begin{array}{l}\text { Some college, } \\
\text { but no degree }\end{array}$ & $9(12.9 \%)$ \\
\hline First born & $36(51.4 \%)$ & & \\
\hline Second born & $23(32.9 \%)$ & & \\
\hline Third born & $8(11.4 \%)$ & & \\
\hline Fourth born & $2(2.9 \%)$ & & \\
\hline Sixth born & $1(1.4 \%)$ & & \\
\hline $\begin{array}{l}\text { Maternal } \\
\text { characteristics }\end{array}$ & & $\begin{array}{l}\text { Associate's } \\
\text { degree }\end{array}$ & $3(4.3 \%)$ \\
\hline $\begin{array}{l}\text { Latina/Chicana/Hispanic, } \\
n\end{array}$ & 30 & $\begin{array}{l}\text { Bachelor's } \\
\text { degree }\end{array}$ & $18(25.7 \%)$ \\
\hline $\begin{array}{l}\text { Cohabiting with baby's } \\
\text { father, } n \text {, yes }\end{array}$ & 70,63 & $\begin{array}{l}\text { Other graduate } \\
\text { degree }\end{array}$ & $16(22.9 \%)$ \\
\hline
\end{tabular}




\begin{tabular}{lll}
\hline & & $\begin{array}{l}\text { (Master's, } \\
\text { Doctorate) }\end{array}$ \\
$\begin{array}{l}\text { Income to needs ratio }(n, \\
\text { mean } \pm S D, \text { median) }\end{array}$ & $\begin{array}{l}\text { Certificate } \\
489.35,364\end{array}$ & $3(4.3 \%)$ \\
$\begin{array}{l}\text { Average years of } \\
\text { education, }(n, \text { mean } \pm\end{array}$ & $68,15.67 \pm$ & \\
$S D)$ & 3.28 & \\
& & \\
\hline
\end{tabular}

Table 3-2. Descriptive statistics for the Bayley Cognitive, Motor, and Language Composite scores at six and 12 months of age.

\begin{tabular}{|c|c|c|c|c|c|c|}
\hline Scale & $\begin{array}{l}\text { Time } \\
\text { (month) }\end{array}$ & $\mathbf{N}$ & Mean & Minimum & Maximum & SD \\
\hline $\begin{array}{l}\text { Cognitive } \\
\text { Composite }\end{array}$ & 6 & 60 & 102.83 & 75 & 120 & 10.27 \\
\hline $\begin{array}{l}\text { Cognitive } \\
\text { Composite }\end{array}$ & 12 & 61 & 105.66 & 85 & 135 & 11.20 \\
\hline $\begin{array}{l}\text { Motor } \\
\text { Composite }\end{array}$ & 6 & 61 & 93.61 & 65 & 115 & 10.69 \\
\hline $\begin{array}{l}\text { Motor } \\
\text { Composite }\end{array}$ & 12 & 61 & 96.40 & 73 & 121 & 10.00 \\
\hline $\begin{array}{l}\text { Language } \\
\text { Composite }\end{array}$ & 6 & 61 & 97.62 & 77 & 115 & 9.02 \\
\hline $\begin{array}{l}\text { Language } \\
\text { Composite }\end{array}$ & 12 & 61 & 99.66 & 77 & 132 & 10.00 \\
\hline
\end{tabular}

Table 3-3. Descriptive statistics for the select fatty acid composition of human milk $(n=70)$ reported as relative percentages.

\begin{tabular}{rrrrr}
\hline Fatty Acid & \multicolumn{1}{c}{ Mean } & Minimum & Maximum & SD \\
\hline DHA & 0.729 & 0.2 & 2 & 0.423 \\
EPA & 0.089 & 0 & 0.3 & 0.075 \\
ARA & 0.883 & 0.5 & 2 & 0.279 \\
ALA & 1.414 & 0.5 & 3.7 & 0.517 \\
LA & 14.569 & 6.9 & 28.4 & 3.257 \\
OA & 35.830 & 25.1 & 47.9 & 4.185 \\
ARA/LA & 0.063 & 0.024 & 0.17 & 0.025 \\
DHA+EPA & 0.817 & 0.2 & 2.3 & 0.482 \\
(DHA+EPA)/ALA & 0.676 & 0.125 & 3 & 0.542 \\
\hline
\end{tabular}


Table 3-4. Linear regression analysis for fatty acids and BSID-III scores. Simple linear regression tables comparing fatty acids and (A) Bayley Cognitive Composite, (B) Motor Composite, and (C) Language Composite scores at six and 12 months of age. Statistically significant correlations $(\mathrm{p}<0.05)$ are denoted with an asterisk $(*)$. B represents the unstandardized coefficient while $\beta$ represents the standardized coefficient.

\begin{tabular}{lllllllll}
\hline A) & \multicolumn{3}{c}{ 6 Months } & \multicolumn{5}{c}{ 12 Months } \\
\hline & $\mathbf{R}^{\mathbf{2}}$ & $\mathbf{B}$ & $\boldsymbol{\beta}$ & $\mathbf{p}$ & $\mathbf{R}^{\mathbf{2}}$ & $\mathbf{B}$ & $\boldsymbol{\beta}$ & $\mathbf{p}$ \\
\hline DHA & 0.004 & 3.498 & 0.144 & 0.274 & -0.006 & 2.913 & 0.103 & 0.428 \\
EPA & 0.030 & 30.769 & 0.215 & 0.099 & -0.016 & 4.926 & 0.032 & 0.809 \\
ARA & 0.005 & -5.246 & -0.147 & 0.263 & 0.057 & -10.498 & -0.269 & $0.036^{*}$ \\
ALA & -0.007 & 2.246 & 0.099 & 0.453 & -0.016 & -0.606 & -0.025 & 0.850 \\
LA & -0.014 & 0.198 & 0.054 & 0.682 & -0.015 & -0.179 & -0.045 & 0.731 \\
OA & 0.031 & -0.552 & -0.218 & 0.094 & -0.017 & 0.054 & 0.020 & 0.880 \\
ARA/LA & -0.015 & 25.959 & 0.051 & 0.701 & 0.036 & -101.629 & -0.228 & 0.077 \\
DHA+EPA & 0.009 & 3.443 & 0.160 & 0.223 & -0.008 & 2.371 & 0.096 & 0.463 \\
(DHA+EPA)/ALA & 0.010 & 3.184 & 0.163 & 0.214 & 0.005 & 3.223 & 0.146 & 0.260 \\
& & & & & & & & \\
\hline
\end{tabular}

\begin{tabular}{lllllllll}
\hline B) & \multicolumn{9}{c}{$\mathbf{6}$ Months } & \multicolumn{5}{c}{$\mathbf{1 2}$ Months } \\
\hline & $\mathbf{R}^{\mathbf{2}}$ & $\mathbf{B}$ & $\boldsymbol{\beta}$ & $\mathbf{p}$ & $\mathbf{R}^{\mathbf{2}}$ & $\mathbf{B}$ & $\boldsymbol{\beta}$ & $\mathbf{p}$ \\
\hline DHA & -0.017 & -0.162 & -0.005 & 0.968 & -0.006 & -1.586 & -0.063 & 0.629 \\
EPA & -0.017 & -1.152 & -0.006 & 0.961 & -0.003 & -16.388 & -0.118 & 0.366 \\
ARA & 0.044 & -11.23 & -0.246 & 0.056 & 0.018 & -6.455 & -0.185 & 0.153 \\
ALA & -0.015 & -1.395 & -0.048 & 0.712 & 0 & -2.798 & -0.128 & 0.327 \\
LA & -0.012 & -0.330 & -0.070 & 0.590 & -0.007 & -0.345 & -0.097 & 0.457 \\
OA & -0.016 & 0.121 & 0.037 & 0.776 & -0.014 & -0.121 & -0.050 & 0.703 \\
ARA/LA & -0.015 & 29.135 & 0.044 & 0.734 & -0.012 & -27.34 & -0.069 & 0.598 \\
DHA+EPA & -0.017 & -0.154 & -0.006 & 0.966 & -0.011 & -1.636 & -0.074 & 0.571 \\
(DHA+EPA)/ & -0.001 & 3.145 & 0.126 & 0.333 & -0.005 & 2.134 & 0.109 & 0.404 \\
ALA & & & & & & & & \\
\hline
\end{tabular}




\begin{tabular}{lllllllll}
\hline C) & \multicolumn{4}{c}{ 6 Months } & \multicolumn{5}{c}{ 12 Months } \\
\hline & $\mathbf{R}^{\mathbf{2}}$ & $\mathbf{B}$ & $\boldsymbol{\beta}$ & $\mathbf{p}$ & $\mathbf{R}^{\mathbf{2}}$ & $\mathbf{B}$ & $\boldsymbol{\beta}$ & $\mathbf{p}$ \\
\hline DHA & 0.002 & 3.456 & 0.136 & 0.297 & 0.01 & -4.063 & -0.161 & 0.214 \\
EPA & 0.041 & 35.359 & 0.238 & 0.065 & -0.016 & -16.388 & -0.118 & 0.366 \\
ARA & 0.034 & -8.392 & -0.224 & 0.083 & 0.050 & -8.940 & -0.256 & $0.046^{*}$ \\
ALA & 0.021 & 4.601 & 0.194 & 0.135 & -0.015 & -2.798 & -0.128 & 0.327 \\
LA & -0.007 & 0.387 & 0.100 & 0.441 & -0.015 & 0.172 & 0.048 & 0.712 \\
OA & -0.008 & 0.253 & 0.095 & 0.466 & -0.008 & 0.235 & 0.096 & 0.460 \\
ARA/LA & -0.011 & -39.464 & -0.073 & 0.574 & -0.004 & 44.851 & 0.113 & 0.387 \\
DHA+EPA & 0.008 & 3.517 & 0.156 & 0.229 & 0.002 & -3.034 & -0.137 & 0.292 \\
(DHA+EPA)/ & -0.015 & 0.858 & 0.042 & 0.748 & -0.009 & -1.786 & -0.091 & 0.486 \\
ALA & & & & & & & & \\
\hline
\end{tabular}

Table 3-5. Regression analysis of ARA and Bayley Cognitive Composite and Language Composite scores at 12 months of age after adjusting for the following covariates: Self-reported years of maternal education, maternal age, INR, ethnicity

\begin{tabular}{lllllll}
\hline & \multicolumn{1}{c}{$\begin{array}{c}\text { Cognitive 12 Months } \\
\text { B }\end{array}$} & $\boldsymbol{\beta}$ & $\mathbf{p}$ & $\mathbf{B}$ & $\boldsymbol{\beta}$ & $\mathbf{p}$ \\
\hline ARA & -10.282 & -0.266 & 0.048 & -8.145 & -0.235 & 0.083 \\
\hline
\end{tabular}

Table 3-6. Linear regression model of ARA in relation to Bayley Cognitive Composite scores at 12 months of age after incorporating the following modifier: Percentage of infant diet comprised of human milk at six months.

\begin{tabular}{llll}
\hline & B & $\boldsymbol{\beta}$ & p \\
\hline (ARA)*(\% Milk) & -0.073 & -0.088 & 0.571 \\
ARA & -9.556 & -0.258 & 0.11 \\
\% Milk & 0.014 & 0.054 & 0.697 \\
\hline
\end{tabular}

The participants in the present study consisted of 70 mothers recruited in Orange County, CA (USA). Demographic information was obtained (Table 3-1), and descriptive statistics were 
calculated for BSID-III scores of the infants (Table 3-2) and for the fatty acid composition of human milk from participants (Table 3-3). At six months, the relation between the individual fatty acids, fatty acid ratios, and BSID-III Cognitive Motor, and Language Composite scores was not statistically significant (Table 3-4A-C). However, the relative percentage of ARA was significantly correlated $(\mathrm{p}<0.05)$ with Bayley Cognitive Composite scores at 12 months of age (Table 3-4A). After including the following covariates in the regression model, this relation remained statistically significant: maternal age, education, ethnicity, and INR (Table 3-5).

Prior to accounting for covariates, ARA was significantly correlated to Bayley Language Composite scores at 12 months of age (Table 3-4C). However, after including covariates in the regression model, this relation became a non-significant trend $(\mathrm{p}>0.05<0.10)$ (Table $3-4 \mathrm{C})$. The following non-significant trends were also observed in the simple linear regression models: the relation between ARA and Bayley Cognitive Composite scores at six months, OA and Bayley Cognitive Composite scores at six months, ARA/LA and Bayley Cognitive Composite scores at 12 months, EPA and Bayley Language Composite scores at six months, and ARA and Bayley Language Composite scores at six months.

Potential modifiers based on the exclusivity of human milk consumption were also considered (Table 3-6). At six months of age, 63 participants out of 70 reported the percentage of infant diet that was comprised of their human milk (data not shown). It was found, however, that the percent of diet from human milk at six months did not moderate the association between ARA and Bayley Cognitive Composite scores (Table 3-6) (all p values $>0.05$ ). 


\section{Discussion}

The relative amount of ALA, ARA, and EPA in milk of participants in the present study (Table 3-3) is similar to values reported in literature (Barreiro et al., 2018). An analysis of the fatty acids in milk from women in Northwest Spain at was similar to milk in the rest of Europe and the world at the same or similar points of lactation (Barreiro et al., 2018). However, several fatty acids quantified for the present study at two weeks of lactation differed from the values from one month of lactation reported by (Barreiro et al., 2018). In the present study, milk from participants had a greater mean concentration of DHA $(0.73 \%)$ than participants in previously reported studies $(0.36 \%)$ (Barreiro et al., 2018). Additionally, milk from participants in the present study contained a mean concentration of ARA almost double that of a previously reported study $(0.883 \%$ and $0.49 \%$ respectively) (Barreiro et al., 2018).

Table 3-1. Average fatty acid content of human milk at one month postpartum in mothers from Northwestern Spain (Barreiro et al., 2018) compared to the fatty acid content of human milk in the present study.

\begin{tabular}{lll}
\hline Fatty Acid & $\begin{array}{l}\text { Average Relative } \\
\text { Percentage (Barreiro et } \\
\text { al., 2018) (n=157) }\end{array}$ & $\begin{array}{l}\text { Average Relative } \\
\text { Percentage in the } \\
\text { Present Study }(\mathbf{n}=\mathbf{7 0})\end{array}$ \\
\hline DHA & $0.36 \pm 0.17$ & $0.729 \pm 0.423$ \\
EPA & $0.11 \pm 0.05$ & $0.089 \pm 0.075$ \\
ARA & $0.49 \pm 0.11$ & $0.883 \pm 0.279$ \\
ALA & $0.48 \pm 0.12$ & $1.414 \pm 0.517$ \\
LA & $14.12 \pm 3.16$ & $14.569 \pm 3.257$ \\
OA & $34.3 \pm 2.61$ & $35.83 \pm 4.185$ \\
\hline
\end{tabular}

Human milk from two weeks and one month postpartum are comparable for research purposes, as after two weeks, milk is considered generally mature (Ballard \& Morrow, 2013). 
Thirty of the 70 participants in the study were Hispanic/Latina (Table 3-1A). However, it is unlikely that elevated DHA composition in the milk obtained from participants of the present study is related to ethnicity. In fact, the fatty acid concentration of DHA in the milk of women in other areas of the world, such as Latin America, was found to be similar to fatty acid profiles from Europe (Barreiro et al., 2018). Thus, the increased concentration of DHA in the milk of women in the present study is likely diet-related. However, maternal dietary records were not obtained to confirm this.

ARA was inversely related to Bayley Cognitive Composite scores at 12 , but not six months of age (Table 3-4;Table 3-6). The importance of this observation extends beyond infancy, since Bayley Cognitive Composite scores in infancy are an indicator of cognitive development in childhood (Berk, 2013). In the present study, the composition of human milk at two weeks postpartum predicted BSID-III Cognitive development at 12 months. The lack of statistically significant findings at six months of age may be attributed to greater accuracy of the health and behavioral assessments at 12 months of age, when infants are more emotive and expressive than at six months of age. In fact, one critique of the BSID-III is that the reliability is lacking for younger children (aged zero to six months). However, reliability is an inherent challenge in any instrument intended for use in populations with high developmental variation, such as young, rapidly developing infants.

In the present study, DHA was not significantly correlated with developmental outcomes at six or 12 months of age. It was previously found that infants whose mothers supplemented with DHA during breastfeeding exhibited a higher Bayley-II Psychomotor Index (predecessor to BSID-III Motor Composite) at 30 months of age, but not at any 12 months of age (Jensen et al., 2005). However, the advantage of a higher Psychomotor Index was unclear, since the mean of all 
groups was above the expected mean. The delayed effect of psychomotor development may have been observed because DHA early in life is necessary for developmental domains that are manifested later in life, or because DHA confers psychomotor benefits that are not detected due to the lack of sensitive tests early in life (Jensen et al., 2005). Therefore, it is possible that DHA or another fatty acid in the present study may have been significantly correlated to Bayley Motor scores if results past 12 months were recorded.

The amount of variation in BSID-III scores explained by fatty acids and fatty acid ratios in statistically significant regression models is smaller than $\mathrm{R}^{2}=0.1$, and can thus be classified as “small," (Murphy \& Myors, 2004) (Table 3-4;Table 3-6). However, a single fatty acid or fatty acid ratio explaining even $5-10 \%$ of the variation in complex development outcomes influenced by other factors such as genetics could have major public health implications, including personalized nutritional recommendations for lactating mothers, and the development of milk fortifiers and infant formulas. Although mothers cannot change genetic factors that may influence the composition of their milk, nutrition is a more accessible and easily-implemented strategy to optimize the nutritional benefits of human milk consumed by infants.

In the present study, ARA was inversely correlated with Bayley Cognitive Composite scores at 12 months after covariate analysis. Relative percentages of other essential fatty acids to which developmental benefits have previously been attributed, such as DHA, did not correlate with BSID-III outcomes. However, this may be due to the specific population in the present study. The average relative percentage of DHA reported in the present study was $0.729 \%$ compared to $0.36 \%$ reported in a previous study at a similar timepoint (Table 3-3). The majority of the infants in the present study may therefore have consumed DHA in excess of levels necessary to confer health and developmental benefits. Hence, variations in the levels of DHA in 
the human milk studied may not have predicted developmental outcomes due to the absence of samples containing little to no DHA. Future work studying human milk with more of a range of DHA concentrations and the ratio of DHA/ARA may provide more insight. It should also be noted that the sample size calculation (Equation 1) does not include the incorporation of covariates. Given the rigorous incorporation of four covariates in the present study, a larger study group may yield statistically significant results in Bayley Language Composite scores after covariate analysis.

The average relative percentage of ARA in the milk of participants in the present study was roughly twice as high as previously reported (Table 3-7). ARA has been associated with cognitive development in infants, and is typically added to infant formula in a 2:1 ratio with DHA in the United States (Lien et al., 2018). In the present study, the ratio of ARA to DHA was 1.1 to 1 (Table 3-3). Interestingly, previous work reported a reduced benefit in some cognitive measures when infants consumed formula with a higher DHA to ARA ratio (1.5:1) compared to intermediate doses of DHA (1:1 and 1:1.5 DHA to ARA). This effect was observed at 60 and 72 months using the Peabody Picture Vocabulary Test, $3^{\text {rd }}$ edition and the Full Scale IQ derived from the Wechsler Preschool and Primary Scale of Intelligence, $3^{\text {rd }}$ edition respectively (Colombo, Shaddy, Kerling, Gustafson, \& Carlson, 2017). Colombo et al. (2017) noted that previous research of LCPUFA distribution in the brain tissue of baboons displayed a similar trend. Baboons that were fed the highest dose of DHA:ARA (also a 1.5:1 DHA:ARA ratio) in formula indicated a reduction in the tissue concentration of ARA in all areas of the brain. By studying the fatty acid composition of red blood cells in the infants, it was determined that the DHA composition of red blood cells at 4 and 12 months of age plateaued between infants consuming the intermediate and high doses of DHA:ARA, indicating saturation at the highest 
dose. Increased concentrations of specific fatty acids in human milk and formula may therefore lead to lower accretion of other fatty acids in areas of the brain, resulting in the reduction in cognitive benefits. Additionally, similar to the findings of the brain tissues in baboons, the concentration of ARA in red blood cells actually decreased in response to the highest dose of DHA compared to the intermediate doses (Colombo et al., 2017).

It was previously hypothesized that a high ratio of omega- 3 to omega- 6 fatty acids led to the inhibition of $\Delta 6$-desaturase, impeding the conversion of LA to ARA, making ARA unavailable for growth (Hadley, Ryan, Forsyth, Gautier, \& Salem, 2016). In the present study, however, the concentration of both DHA and ARA were higher than previously found, so this may not be a plausible explanation for the inverse correlation between ALA and infant development. Additionally, due to low conversion rate of LA to ARA, the levels of these fatty acids in human milk are primarily reflective of the preformed DHA and ARA consumed in diet. Thus, the mechanistic relation between fatty acids, such as ARA, and developmental outcomes is exceedingly complex, and should consider relative percentages of specific fatty acids interacting in the complex milk matrix.

Adequate concentrations of PUFAs in the brain early in life are important, because fatty acids influence processes such as membrane dynamics and cell signaling. As the infant's brain grows, levels of ARA and DHA increase during the last three months of pregnancy and during the first five years of life. Importantly, dietary intake of fatty acids early in life affects fatty acid accretion in the brain. In contrast to the cerebral cortex, which is of interest for higher-level brain functioning, the cerebellum is important for movement and balance. The percentage of DHA in white matter in the cerebellum has been found to be lower in formula fed infants than in infants fed human milk. However, no differences in cerebellar white matter content were observed for 
ARA (Lien et al., 2018). EFA are thought to promote development through a variety of mechanisms. While DHA is understood to control signaling membranes in the brain and nervous system, ARA is important for brain growth during gestation and early in infancy, where it plays a role in cell signaling and division. ARA mediates various functions in the brain, including neuronal firing, signaling, and long-term potentiation. Among other functions, ARA also maintains membrane order and hippocampal plasticity (Hadley et al., 2016).

Understanding accretion of fatty acid levels in areas of the brain associated with specific functions may mechanistically implicate the role of specific fatty acids in different development functions. Moreover, quantifying the proportion of fatty acids necessary for optimal development may lead to greater understanding of structure-function relationships. This knowledge may also facilitate application of this knowledge to nutritional recommendations and development of milk fortifier and infant formula products. The present study represents novel research, because specific percentages of ARA present in human milk, instead of infant formula, have now been quantified and correlated to BSID-III Cognitive, Motor, and Language scores. BSID-III outcomes can be extrapolated more broadly to cognitive, motor, and language ability in infants. Objectively quantifying and relating fatty acids in human milk to development is necessary to develop nutritional guidelines for breastfeeding mothers and to inform the development of infant nutrition products. Quantitative knowledge implicating specific percentages of product to precursor fatty acids in relation to development is also a segue to future work considering the FADS gene and maternal and infant genotype. 


\section{Limitations}

The present study has several limitations. The gold standard of milk collection involves sampling all milk expressed over 24 hours (Ballard \& Morrow, 2013). This was not the practice for the present study. In addition to limitations related to the collection of milk aliquots, the scope of the present study also presents a limitation. Maternal and infant genotyping was not performed, so genotype could not be incorporated into the regression models as a modifier. It is well known that there is inter-individual variability in the metabolism of fatty acids. The FADS-1 and FADS-2 enzymes encoded by the FADS gene modulate the synthesis of ARA and DHA from LA and ALA respectively. Polymorphisms in the FADS-1 and FADS-2 genes encode the enzymes that alter the production and thus concentration of LC-PUFAs (Lattka et al., 2009). In future work, controlling for how efficiently lactating mothers can synthesize LC-PUFAs that are present in milk and how efficiently infants can synthesize LC-PUFAs from the precursors in the milk they consume will provide a more objective measure of the developmental outcomes associated with specific essential fatty acids. An additional limitation of the present study is that maternal diet and supplementation records were not obtained. For a more comprehensive analysis, future work could include maternal diet and supplementation records in addition to infant and maternal genotyping.

\section{Conclusion}

In conclusion, the relative percentage of ARA in human milk may influence language and cognitive ability in infants. There was an inverse relation between ARA and Bayley Cognitive Composite scores at 12 months of age, and an inverse relation (non-significant trend) between ARA and Language Composite scores 12 months of age. Exclusivity of human milk feeding at six months did not modify BSID-III outcomes in the study population. Future work with a larger sample population may yield additional statistically significant results after accounting for 
covariates. Furthermore, future work accounting for other known modifiers, such as maternal and infant FADS genotype, may provide a more thorough understanding of the relation between essential fatty acids and development. The statistically significant correlations and nonsignificant trends observed in present study prompt further consideration of optimal proportions of fatty acids in human milk and infant formula to inform nutritional recommendations for breastfeeding mothers and product development initiatives. 


\section{Isolation of EVs from Human Milk}

\section{Introduction}

It is well-known that consumption of human milk is associated with enhanced infant health outcomes in comparison to consumption of infant formula. However, it is not fully known which components of human milk may be responsible for supporting optimal health and development of newborns. Increasing research suggests that EVs from human milk have physiologic function that may impact acute and chronic health outcomes. Human milk EVs promote epithelial cell growth in the intestine (Hock et al., 2017a) and were found to protect intestinal epithelial cells from oxidative stress (C. Martin, Patel, Williams, Arora, \& Sims, 2018). Additionally, human milk EVs have been implicated in the immune modulating function of human milk, and may play a role in the development of the neonatal immune system (Zonneveld et al., 2014). These effects may be attributed to the protein, lipid, or microRNA cargo of human milk EVs (Zonneveld et al., 2014). A reliable method for consistent isolation of EVs from human milk is needed to determine the functional components of EVs to which enhanced infant health outcomes can be attributed.

Although UC is the most commonly used method to isolate EVs from biospecimens ( $\mathrm{T}$ Skotland et al., 2017), the feasibility of this method for human milk research is limited. As a precious biofluid for feeding newborns, acquiring the large volume of human milk needed for $\mathrm{EV}$ isolation using $\mathrm{UC}$ is not always feasible. Unfortunately, no method of EV isolation has been authenticated for use with a low volume of human milk $(\leq 2 \mathrm{ml})$; prior studies have isolated human milk EVs from a starting volume of $9 \mathrm{~mL}$ (Vaswani, Qin Koh, Almughlliq, Peiris, \& Mitchell, 2017). As a result, the limitations in conducting research in this area have created a knowledge gap. Additionally, authentication of an EV isolation method from low volumes of 
human milk will facilitate research on EVs throughout milk production periods, the course of lactation, over time-of-day variation, and perhaps most importantly in low volume producers, which are not adequately studied.

This limitation has several potential negative consequences. First, analysis of only large volumes may limit research to use of pooled human milk. This measure would result in a greater understanding of average milk composition but not of interindividual variability. Second, analyses may be limited solely to time of lactation when higher volumes of milk are produced. This may then result in a disparate understanding of mature milk relative to early and transitional milks. Finally, research may be limited to studies of mothers with high volume of milk expression instead of low volume producers. Therefore, a strong need exists for a method of isolate EVs from a low volume of human milk.

A novel method for the isolation of EVs requires verification procedures. The International Society for Extracellular Vesicles (ISEV) released MISEV guidelines in 2018 (Théry, Witwer, \& Aikawa, 2018) detailing the minimum criteria for confirming isolation of EVs. MISEV guidelines recommend that each EV preparation be (i) defined quantitatively by the source of EVs, (ii) characterized to determine the abundance of EVs by total particle number or protein/lipid content, (iii) tested for components associated with EV subtypes or EVs generically, and (iv) tested for the presence of non-vesicular co-isolated components. This paper describes a precipitation-based method for the isolation of EVs from human milk. The subsequent characterization of EVs suggest successful isolation in compliance with the MISEV guidelines. EVs isolated using the present method are therefore appropriate for downstream characterization and functional analyses to better understand the health and immune-modulating properties of human milk. 


\section{Methods}

Mature milk ( $>6$ months after initiation of lactation) was pooled and pasteurized from donors to develop the EV isolation method (Prolacta Bioscience, City of Industry CA). Twelve volunteers also provided samples of expressed milk between 2 and 4 weeks postpartum which were immediately frozen at $-80^{\circ} \mathrm{C}$. Ethics approval was obtained from the Chapman University Institutional Review Board. The EV isolation method (Figure 4-1) outlined herein is adapted from the instructions for a commercial precipitation reagent (SBI, 2018b) and a previously published protocol (X. Wang, 2017).

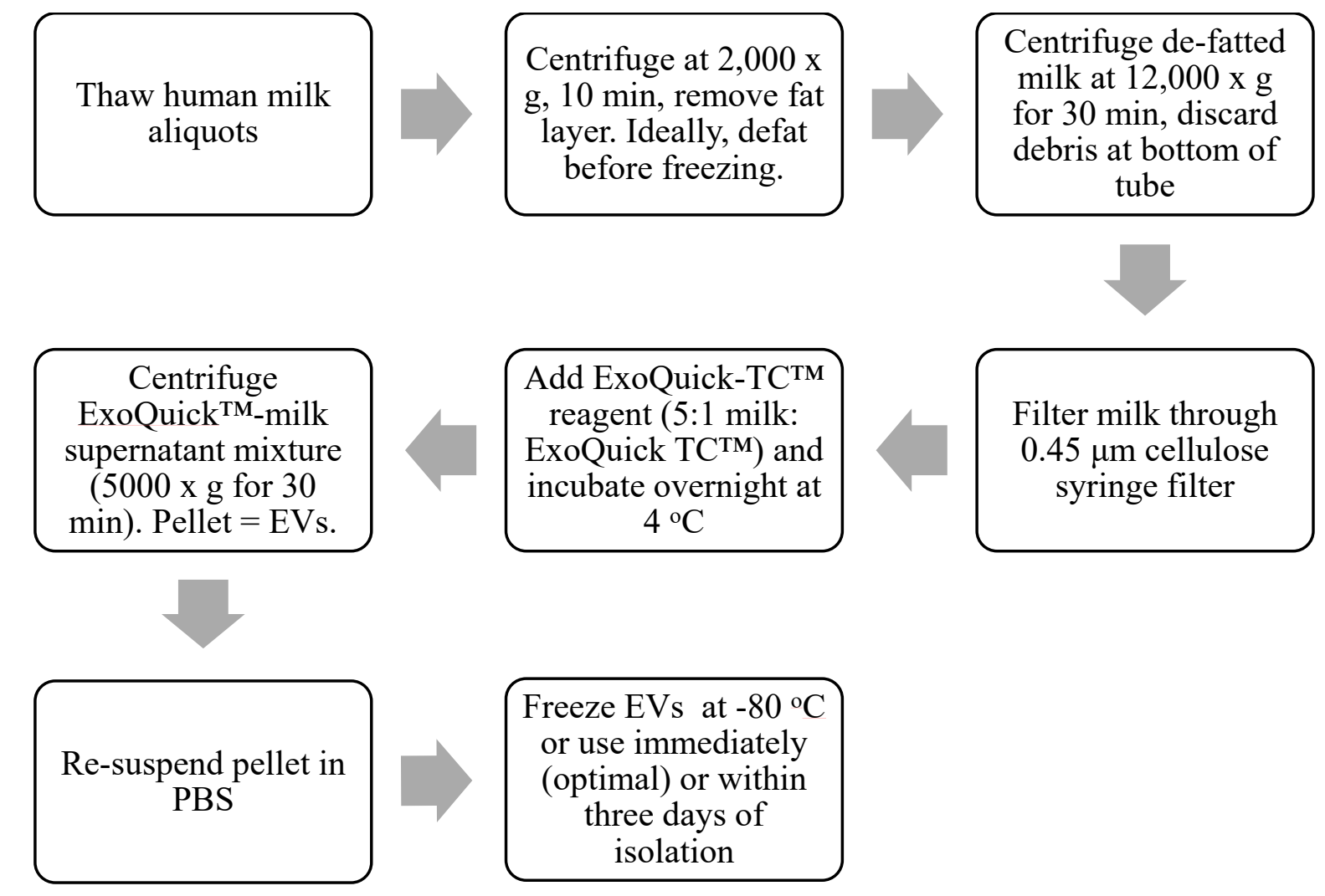

Figure 4-1. Workflow schematic for EV isolation using a precipitation-based isolation method. 


\section{EV Isolation Method}

\section{Thawing, Defatting, and Removal of Cell Debris}

1. Thaw frozen human milk at $4{ }^{\circ} \mathrm{C}$. Once thawed, vortex milk for $\sim 3 \mathrm{~s}$.

2. If milk was not aliquoted into microcentrifuge tubes prior to freezing, aliquot $1.5-2 \mathrm{~mL}$ (or desired volume) human milk into microcentrifuge tubes.

3. Centrifuge at 2,000 $\times \mathrm{g}$ for $10 \mathrm{~min}$ to separate and remove the fat layer with a metal spatula. Discard the fat layer and transfer milk to a new tube. Removing the fat layer also removes milk fat globules (Le et al., 2009).

4. Centrifuge the defatted milk at $12,000 \times \mathrm{g}$ for $30 \mathrm{~min}$ to remove cell debris. Transfer milk supernatant and/or discard pellet.

5. Filter milk supernatant through a $0.45 \mu \mathrm{m}$ cellulose syringe filter into a new microcentrifuge tube to further eliminate cells and cellular debris.

\section{EV Isolation}

6. Using a 5:1 ratio of milk supernatant: ExoOuick-TC ${ }^{\text {TM }}$ reagent (Svstem Biosciences. Palo Alto CA). add reagent to the filtered milk and gentlv invert until mixed.

7. Incubate at $4^{\circ} \mathrm{C}$ overnight or for at least $12 \mathrm{~h}$.

8. After incubation, centrifuge at $5.000 \times \mathrm{g}$ for $30 \mathrm{~min}$ (beige pellet will appear at the bottom of the tube).

9. Discard supernatant. and resuspend EV pellet in 100-600 uL PBS (pH 7.4).

10. Depending on downstream application. use resuspended EVs stored at $4{ }^{\circ} \mathrm{C}$ within 3 davs or freeze immediately at $-80^{\circ} \mathrm{C}$.

\section{Scanning Electron Microscopy}

Zeiss Gemini Sigma 300 scanning electron microscope (SEM) was used to visualize EVs isolated from milk expressed at 2 weeks postpartum ( $n=1$ volunteer). EVs were visualized 1 day 
after they were isolated, resuspended, and stored at $4^{\circ} \mathrm{C}$. The original $\mathrm{EV}$ resuspension in PBS $(500 \mu \mathrm{L})$ was further diluted in PBS $(1: 1,000)$. SEM slides were prepared with $2 \mu \mathrm{L}$ of diluted EVs. Argon gas sputter coating of EVs with $3 \mathrm{~nm}$ gold-palladium alloy was performed to prevent sample destruction.

\section{Nanoparticle Tracking Analysis}

Nanoparticle Tracking Analysis (NTA; Nanosight NS01) was used to determine the concentration and size of EVs isolated from the pooled milk sample. A sample of EVs originally resuspended in PBS $(500 \mu \mathrm{L})$ and frozen at $-80^{\circ} \mathrm{C}$ was thawed on ice and further diluted in PBS $(1: 75)$ prior to injection. Detection threshold was set to four, and three runs each of $30 \mathrm{~s}$ in duration were completed and analyzed using NTA 3.1 software. Total yield (EV particles/mL milk) was calculated based on dilution factors and a starting volume of $1.5 \mathrm{~mL}$ milk.

\section{Dynamic Light Scattering}

The diameter of EVs isolated from the pooled milk sample was measured with a Mobius Dynamic Light Scattering (DLS) instrument (Wyatt Technology) using DLS Firmware Version 1.2.0.0. Laser wavelength was set to $532 \mathrm{~nm}$, and a detector angle of $163.5^{\circ}$ was used. DLS acquisition time was set to $5 \mathrm{~s}$ and a number acquisition of three was used to perform three technical replications on EVs stored at $4^{\circ} \mathrm{C}$ over the course of 10 days. 


\section{Exocheck Antibody Array}

The Exocheck ${ }^{\mathrm{TM}}$ Antibodv Arrav (Svstem Biosciences. Palo Alto CA) was used according to the manufacturer's instructions (SBI, 2018a) to determine the presence or absence of common EV proteins (CD63, EpCAM, Annexin5, TSG101, Flotilin1, ICAM, ALIX, CD81) in EVs isolated from milk expressed at 4 weeks postpartum ( $n=1$ volunteer). Resuspended EVs were thawed on ice prior to antibody array analysis.

\section{Determination of Total Fatty Acid Concentration}

The EVs from which fatty acids were analyzed were isolated using $2 \mathrm{~mL}$ aliquots of pooled milk, and with variations in EV isolation steps. A 5:1 and 10:1 ratio of milk supernatant: ExoQuick$\mathrm{TC}^{\mathrm{TM}}$ reagent was used with or without $(0.45 \mu \mathrm{m}$ cellulose $)$ filtration or purification using ExoQuick-TCTM ULTRA purification columns according to the manufacturer's instructions (System Biosciences, Palo Alto, CA). Prior to fatty acid analysis, EVs were isolated from the pooled milk sample, resuspended in PBS $(500 \mu \mathrm{L})$, frozen at $-80^{\circ} \mathrm{C}$, and thawed on ice. Fatty acid analysis was performed by Creative Biostructure (Shirley, NY USA).

The total fatty acid concentration of EVs was determined by colorimetric analysis in triplicate ( $n=1$ per isolation variation). Standards were prepared with palmitic acid $(1 \mathrm{nmol} / \mu \mathrm{L})$. Samples were diluted and homogenized. Standard dilution $(50 \mu \mathrm{L})$ or sample $(0.5-25 \mu \mathrm{L})$ were added to each sample well. The final volume was adjusted to $50 \mu \mathrm{L}$ with assay buffer. An acyl-coenzyme A synthetase reagent $(2 \mu \mathrm{L})$ was added to each reaction well, mixed, and incubated (20 min, $\left.37^{\circ} \mathrm{C}\right)$. Samples were then incubated $\left(30 \mathrm{~min}, 37^{\circ} \mathrm{C}\right)$ in the dark with reaction mix $(2 \mu \mathrm{L})$ containing assay buffer $(44 \mu \mathrm{L})$, fatty acid probe $(2 \mu \mathrm{L})$, enzyme mix $(2 \mu \mathrm{L})$, and enhancer $(2$ $\mu \mathrm{L})$. Finally, optical density was measured on a microplate reader at $562 \mathrm{~nm}$. 


\section{Protein Quantification}

A Qubit ${ }^{\mathrm{TM}} 4$ Fluorometer was used to measure the protein concentration in human milk EVs isolated from milk expressed at 2 weeks postpartum ( $n=10$ volunteers). Resuspended EVs were thawed on ice prior to protein quantification. The instrument was calibrated with protein standards according to the manufacturer's instructions (ThermoFisher, 2018). EV samples originally resuspended in $600 \mu \mathrm{L}$ PBS were thawed on ice and diluted in PBS (1:20). Lysis buffer $(10 \mu \mathrm{L})$ was added and samples were vortexed (Protease Inhibitor Cocktail, RIPA buffer, Thermo Fisher Scientific, Waltham MA). Protein concentration was measured in duplicate after incubating (15 min, room temperature) the lysate $(1 \mu \mathrm{L})$ with working reagent $(199 \mu \mathrm{L})$. Protein quantification of EVs was calculated based on dilution factors and a starting volume of $1.5 \mathrm{~mL}$ milk.

\section{Results}

SEM (Figure 4-2), NTA (Figure 4-3) DLS (Figure 4-4), and an antibody array (Figure 4-5) were used to image, quantify, measure the average diameter, and identify protein markers characteristic of EVs. The image obtained by SEM revealed the size of nanovesicles in the expected range for EVs, approximately 50-350 nm (Figure 4-2). Results from analysis by NTA revealed that the isolation method yielded $8.9 \times 10^{9}\left( \pm 1.1 \times 10^{9}\right)$ particles $/ \mathrm{mL}$ of human milk. The mean and mode diameter of EVs were 179.3 and $150.3 \mathrm{~nm}$, respectively (Figure 4-3). No standard deviation is reported for the mean since a trimodal distribution of EV populations was observed. Results from DLS (Figure 4-4) showed that the average diameter of EVs 1 day after isolation was 213.6, and $249.7 \mathrm{~nm} 10$ days after isolation. Error bars for individual days were excluded because individual standard deviations for technical replicates were $<5 \%$ of the mean. Antibody array (Figure 4-5) indicated that the sample was positive for the following known EV 
markers: cluster of differentiation 81 (CD81), ALG-2-interacting Protein X (ALIX), intracellular adhesion molecule (ICAM), tumor susceptibility gene 101 (TSG101), and Annexin5, and negative for cluster of differentiation 63 (CD63), epithelial cell adhesion molecule (EpCAM), and flotilin1.

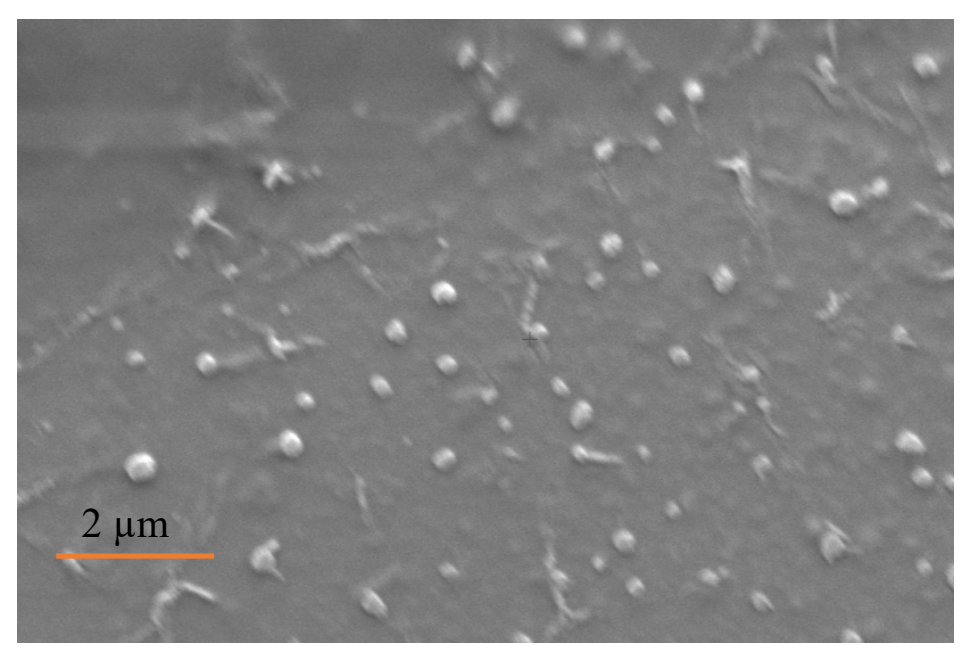

Figure 4-1. Image of EVs obtained by SEM from participant $(\mathrm{n}=1)$, Electron high tension $=$ $5.00 \mathrm{kV}$, working distance $=20.9 \mathrm{~mm}$, detector $=$ secondary electron, magnification $=8.70 \mathrm{~K} \mathrm{X}$, vacuum mode $=$ high vacuum, height $=9.851 \mu \mathrm{m}$. 
$\mathbf{A}$

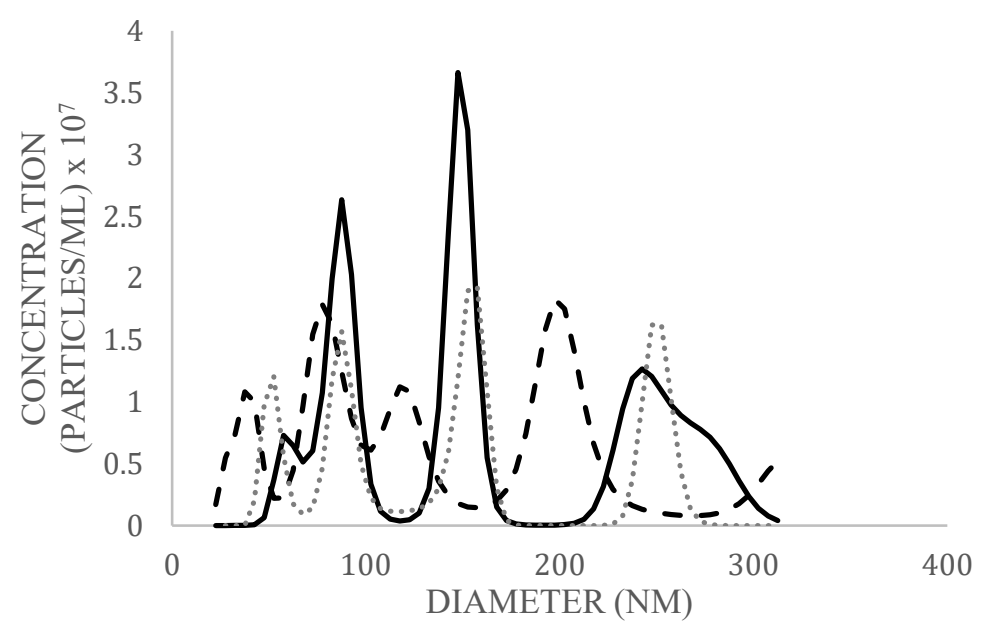

B

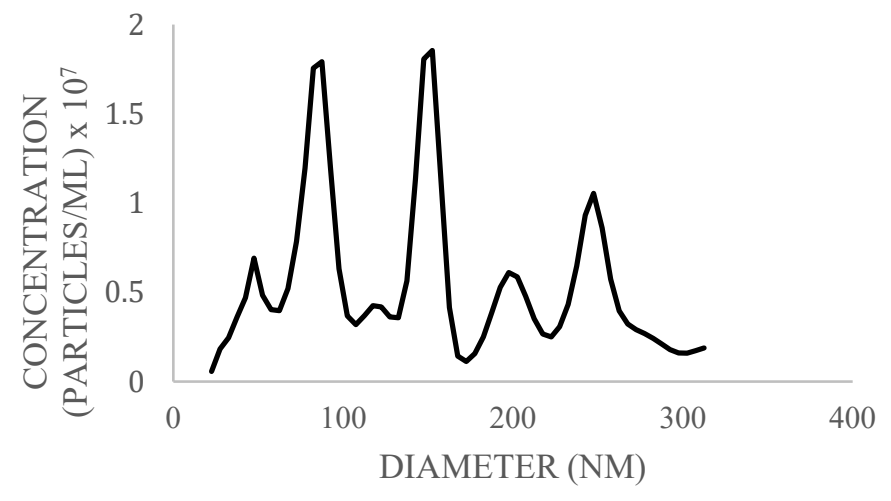

Figure 4-3. Diameter and concentration of human milk EVs from pooled human donor milk measured by NTA. Technical replicates were performed in triplicate (solid line $=$ trial 1 ; dashed line $=$ trial 2 ; dotted line $=$ trial 3 ) (A) and the average of the three runs was calculated (B). The above graphs are plotted from the 10th-90th percentile of EV sizes $(22.5-312.5 \mathrm{~nm})$ to exclude particles which do not meet the size criterion for likely EVs. 


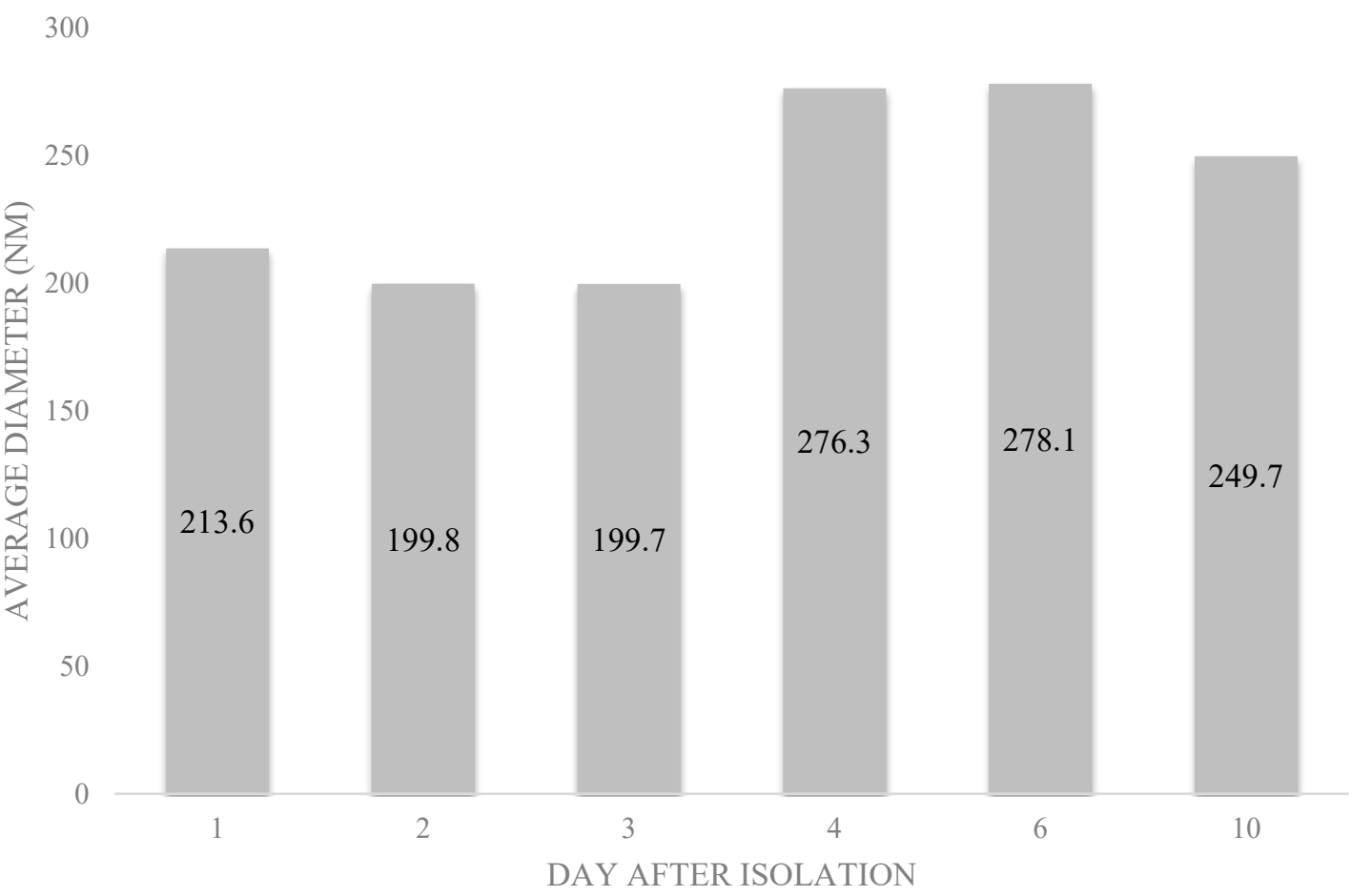

Figure 4-2. Average diameter of EVs from pooled human donor milk measured for 10 consecutive days after isolation and storage at $4^{\circ} \mathrm{C}$. Error bars were excluded because individual standard deviations were $<5 \%$ of the mean. Pooled $\mathrm{SD}=33.45$.

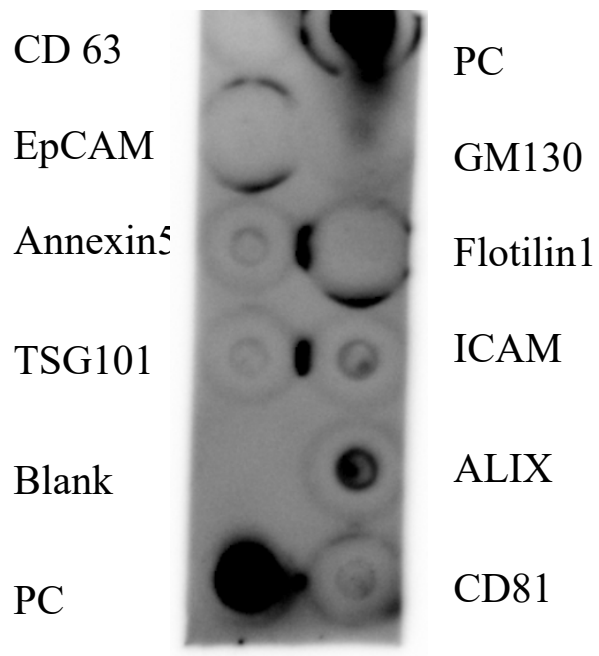

Figure 4-3. Antibody array of human milk EVs from participant $(n=1)$. PC represents the positive control, and GM130 is a cellular contamination marker. CD $63=$ cluster of differentiation 63, EpCAM = epithelial cell adhesion molecule, TSG101 = tumor susceptibility gene 101, ICAM = intracellular adhesion molecule, ALIX = ALG-2-interacting Protein X, CD81 $=$ cluster of differentiation 81 . 
After verification of isolation, human milk EVs were characterized by quantifying total fatty acids (Figure 4-6) and protein concentration (Table 4-1). The average total fatty acid concentration of EVs isolated with the recommended method (5:1, filter, no column purification) was $36.94 \mathrm{mg} / \mathrm{dL}$. The mean protein concentration of human milk EVs was $5.08( \pm 0.15) \mathrm{mg} / \mathrm{dL}$.

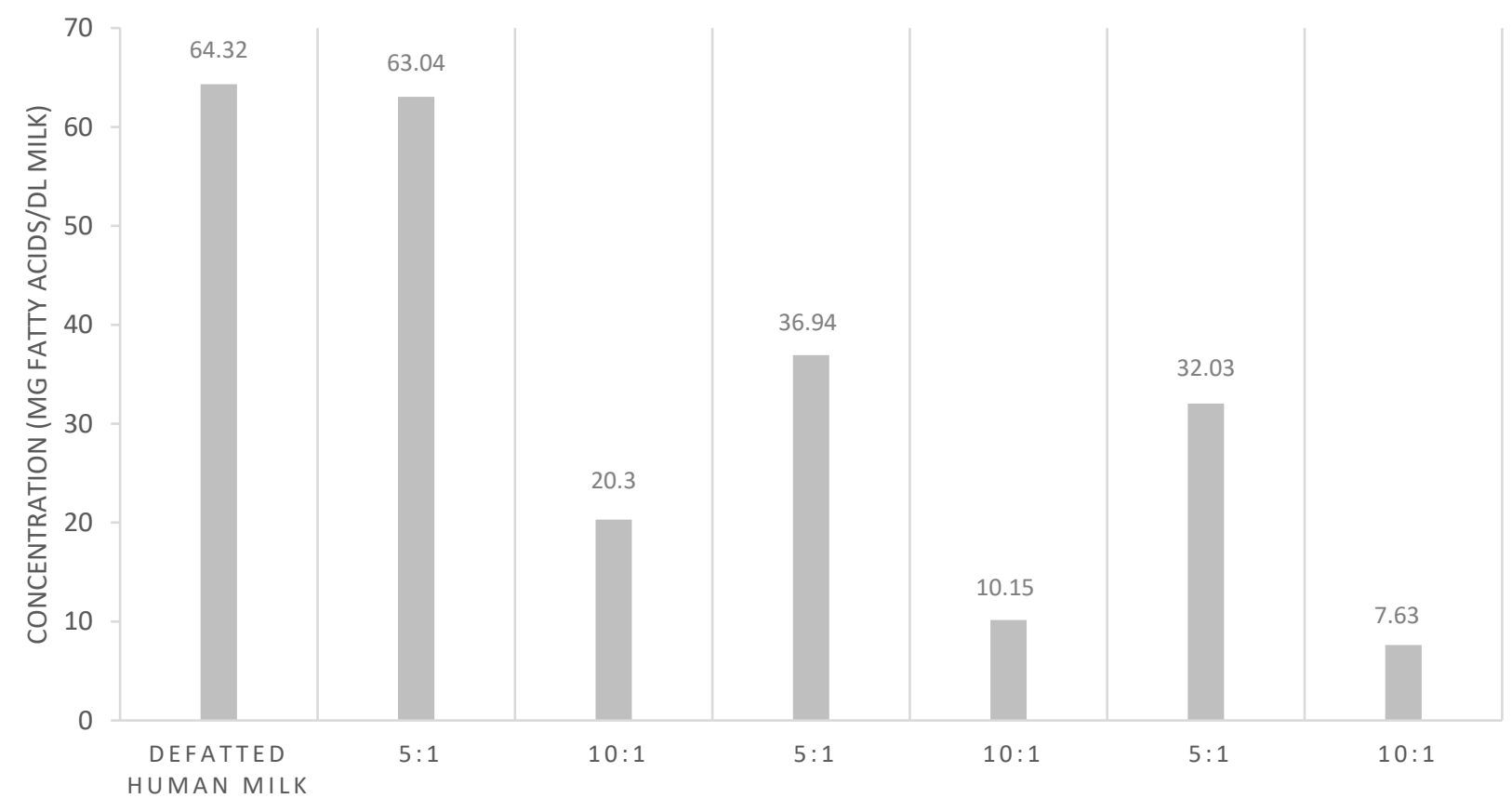
$0.45 \mu \mathrm{m}$ filter $\mathrm{N} / \mathrm{A}$
$(-)$
$(-)$
$(+)$
$(+)$
$(-)$
$(-)$
Column
N/A
$(-)$
$(-)$
$(-)$
$(-)$
$(+)$
$(+)$
affinity
purification

Figure 4-4. Antibody array of human milk EVs from participant $(n=1)$. PC represents the positive control, and GM130 is a cellular contamination marker. CD $63=$ cluster of differentiation 63, EpCAM = epithelial cell adhesion molecule, TSG101 = tumor susceptibility gene 101, ICAM = intracellular adhesion molecule, ALIX = ALG-2-interacting Protein X, CD81 $=$ cluster of differentiation 81 . 


$$
\begin{gathered}
\frac{4.329 \mathrm{nmol} \mathrm{FA}}{\mu \mathrm{L}} \times 500 \mu \mathrm{L} \text { PBS }=2,164.5 \mathrm{nmol} \mathrm{FA} \\
\frac{2,164.5 \mathrm{nmol} \mathrm{FA}}{1.5 \mathrm{~mL}}=\frac{1443 \mathrm{nmol} \mathrm{FA}}{\mathrm{mL} \mathrm{milk}} \\
\frac{0.000000256 \mathrm{~g}}{\mathrm{nmol}} \times \frac{1443 \mathrm{nmol}}{\mathrm{mL}}=\frac{36.94 \mathrm{mg} \mathrm{EV} \mathrm{FA}}{\mathrm{dL} \mathrm{human} \mathrm{milk}}
\end{gathered}
$$

Equation 4-1. Calculation of average concentration of fatty acids present in human milk EVs. Total fatty acids were measured.

Table 4-1. Protein concentration of EVs isolated from human milk of 10 different participants measured in duplicate.

\begin{tabular}{ll}
\hline Sample & Protein content (mg/dL milk) \\
\hline 1 & 5.20 \\
2 & 5.00 \\
3 & 5.11 \\
4 & 5.02 \\
5 & 5.15 \\
6 & 5.18 \\
7 & 5.24 \\
8 & 4.98 \\
9 & 5.20 \\
10 & 4.75 \\
\hline Mean & 5.08 \\
SD & 0.15 \\
\hline
\end{tabular}

\section{Discussion}

The method of EV isolation from human milk described herein meets the MISEV criteria (Théry et al., 2018) for verifying the presence of EVs. EVs isolated with the proposed method were (i) quantified in relation to the source of human milk, (ii) characterized to determine the abundance of EVs by total particle number and lipid \& protein content, (iii) tested for the presence of markers associated with EVs, and (iv) tested for the presence of non-vesicular coisolated components. The method adapted from manufacturer instructions for a precipitation 
reagent (SBI, 2018b) and previous literature (X. Wang, 2017) was shown to be suitable to adequately characterize EVs isolated from human milk and for downstream applications.

There is consistency between the average EV diameter measured by SEM, NTA, and DLS (Figures 4-2, 4-3, and 4-4). Unlike NTA which generates size distribution data, DLS measures the average particle diameter. Measurement by DLS then may be skewed by low concentrations of outliers or clustering of particles (Van der Pol et al., 2010). Therefore, the $\sim 15 \%$ difference in diameter between SEM, NTA, and DLS measurements could be due to overestimation of diameter by DLS. The recommended method presented herein yielded $8.9 \times$ $10^{9}\left( \pm 1.1 \times 10^{9}\right) \mathrm{EV}$ particles $/ \mathrm{mL}$ of human milk. Another group isolated human milk EVs and reported a yield of $8.0 \times 10^{10}$ particles $/ \mathrm{ml}$ of milk using a UC based method (Vaswani et al., 2017). The difference in yield could be attributed to the fact that banked, pasteurized milk was used in the present method. Additionally, EVs were frozen and thawed prior to quantification without defatting before initial freezing, which has shown the decreased recovery of EVs (Zonneveld et al., 2014).

In the MISEV guidelines, it is recommended that operational terminology for extracellular vesicles based on factors such as size be used. EVs $<200 \mathrm{~nm}$ in diameter would be considered "small," and EVs $>200 \mathrm{~nm}$ considered medium or large (Théry et al., 2018). Results from NTA indicated that the greatest concentration of particles is around $153 \mathrm{~nm}$ (Figure 4-3), meaning the EV population in highest abundance would be classified as small. The 10th-90th percentile of particle size were graphed (Figure 4-3) as particles outside this range were likely aggregates or fragments. 
Because storage conditions may affect EV characterization, MISEV guidelines indicate the importance of describing storage conditions such as storage container, temperature, buffer, freeze-thaw cycles of biofluid and EVs, etc. (Théry et al., 2018). It was previously found even that storage of EVs for $2 \mathrm{~h}$ at $4^{\circ} \mathrm{C}$ decreased the viability of the exosome population, but the change in size was not measured (Zonneveld et al., 2014). The timecourse experiment (Figure 44) represents storage-induced changes in diameter starting from freshly isolated EVs measured over the course of 10 days. The average diameter of EVs measured by DLS increased over time after isolation and storage. This may indicate swelling and enlargement of EVs, or aggregation of particles. Therefore, when performing studies to determine the relation between structure and function, it may be advantageous to use EVs immediately after isolation.

For protein-based verification of EV isolation, MISEV guidelines stipulate that at least one type of protein in two broad categories should be positively identified and the absence of one negative marker indicated. These categories include transmembrane or GPI-anchored proteins, such as the tetraspanins CD63 and CD81, and EV-recovered cytosolic proteins such as ALIX and flotillins- 1 and 2. To verify the absence of non-EV isolated co-structures, markers such as albumin can be used (Théry et al., 2018). The antibody array (Figure 4-5) verified that human milk EVs isolated were positive for proteins in the tetraspanin and EV-recovered cytosolic proteins category, and also negative for cellular contamination marker.

The amount of exosomal protein has been used as a means of EV quantification (Kim, Tan, \& Lubman, 2015). Considering that the average protein concentration measured in EVs was $5.08 \mathrm{mg} / \mathrm{dL}, \mathrm{EV}$ protein comprises $\sim 0.42 \%$ of total protein from mature human milk, assuming the protein concentration of mature human milk is $\sim 1,200 \mathrm{mg} / \mathrm{dL}$ (Ballard \& Morrow, 2013). However, it should be noted that protein quantification with biofluids such as human milk may 
not be a consistent and reliable method of quantification due to the presence of co-isolated molecules. Therefore, we reported the total fatty acid concentration of human milk EVs (Figure 4-6). Based on the assumption that fat content of human milk is primarily in the form of triglyceride, we estimated that EV fatty acids are $\sim 0.8 \%$ of total fatty acids in mature human milk (Ballard \& Morrow, 2013).

We compared fatty acid quantification among EVs isolated from human milk with different volumes of reagent, use of size exclusion filter, and with or without column affinity purification. We suggest a supernatant-to-precipitation reagent ratio of 5:1 for optimal yield of EVs to quantify fatty acids. We also suggest filtration of milk by size exclusion after defatting to remove non-EV artifacts such as casein and cellular debris. However, it is unclear whether column affinity purification after EV isolation performs similarly to size exclusion filtration of milk supernatant prior to EV isolation. Although fatty acid quantification was similar after each method, it is unknown if filtration and purification result in differences in the EV populations isolated.

The present method of isolating EVs from human milk fulfills the MISEV criteria by characterizing the EVs with quantitative and qualitative methods, confirming the presence of characteristic EV markers, and confirming (Figure 4-5) the absence of non-EV components. The application of this isolation method extends beyond the applications detailed in our manuscript. The ability to successfully isolate EVs from small volumes of human milk can be applied to miRNA isolation, proteomics, lipidomics, and functional in vitro assays. 


\section{Conclusions}

EVs were successfully isolated from human milk using a precipitation reagent. The method yielded $8.9 \times 10^{9} \pm 1.1 \times 10^{9} \mathrm{EV}$ particles $/ \mathrm{mL}$ of human milk. Protein and fatty acid concentration of EVs in human milk were determined and the percentage of fatty acids and protein in EVs relative to the whole milk were $\sim 0.8 \%$ and $\sim 0.42 \%$, respectively. The method presented is consistent and reliable for isolating, quantifying, and characterizing human milk EVs for research and clinical purposes and in continuing to understand the human milk food matrix. As a dynamic food and biofluid, future study may elucidate how EVs vary over i) early, transitional and mature milk production periods, ii) course of lactation (fore vs. hind milk), and iii) time-of-day variation. This method can be used to elucidate the role of human milk EVs in neonatal health and immune system development, and for applications of formula and human milk fortifier production. 


\section{Fatty acids and EVs in human milk: Global discussion, conclusions, and future work}

\section{Discussion}

The overarching goals of this project were to correlate the fatty acid composition of human milk and human milk EVs to infant development (Cognitive, Behavioral, and Motor) outcomes reported by the Bayley Scales of Infant Development (BSID-III). However, the latter objective was not feasible due to the lack of a suitable method to isolate EVs from small volumes $(<2 \mathrm{~mL})$ of human milk. Thus, in order to carry out this hypothesis, the following objectives were investigated instead: i) whether essential fatty acids correlated to BSID-III developmental outcomes, and ii) whether there was a consistent method to isolate EVs from human milk. Both objectives investigated in the present study were novel and resulted in novel research findings. Specifically, it was found that ARA was inversely correlated to Bayley Cognitive Composite scores, and EVs were able to be successfully isolated from human milk. EV isolation was also validated according to the criteria of the International Society of Extracellular Vesicles (Théry et al., 2018).

The present research is significant, because it represents the possibility of implementing modifiable changes that can confer long term benefits. Many predictors of long term health, such as genetics and socioeconomic status cannot be modified. However, nutrition is unique in that it is a modifiable factor that can be controlled from infancy onwards. Although public health disparities may prevent lactating mothers from accessing recommended nutrition, the present research also pertains to the optimization of commercial infant formula products. Even if parents are unable to provide their child with human milk, or lactating mothers are unable to follow 
nutritional guidelines, associating fatty acids and EVs with infant development will facilitate product development for commercial infant formula products that confer the developmental benefits associated with human milk.

Bioactive compounds in human milk, such as the milk fat globule membrane (MFGM) have already been extensively studied in relation to infant nutrition. MFGM acts as a natural emulsifier to prevent the fat molecules from coalescing in milk and against enzymatic action (Singh, 2006). Various nutritional benefits, such as the reduction of necrotizing enterocolitis and neonatal gut maturation have been associated with MFGM (Dewettinck et al., 2008). MFGM have already been extensively characterized in human milk with respect to the lipid (Demmelmair, Koletzko, \& panel, 2018), and protein (Yang et al., 2020) composition, and have even been incorporated into infant formula (Gallier et al., 2015). However, the relation between the composition of other bioactive compounds in human milk, such as EVs, and infant development remains unknown. Now that a method to isolate EVs from human milk has been validated, this gap in knowledge can be addressed. A consistent method of EV isolation will permit structure and functional analyses, which could eventually facilitate the incorporation of EVs in infant formula products. Overall, the objectives of the present study were met. Clinical research can incorporate this knowledge into product development and nutritional recommendations for babies and mothers. 


\section{Conclusions and Future Work}

The present study examined the relation between bioactive components in human milk and infant developmental outcomes. It was determined if i) essential fatty acids correlated to BSID-III developmental outcomes, and ii) whether there was a consistent method to isolate EVs from human milk. It was found that ARA was inversely correlated with cognitive outcomes at 12 months of age. EVs were successfully isolated from a small $(<2 \mathrm{~mL})$ volume of human milk using an adapted precipitation method, and were subsequently qualitatively and quantitatively characterized. Overall, this work can inform education initiatives to promote optimal infant health and development outcomes through maternal nutrition. Future work could also extend upon the present study to examine EV composition. With the optimization of a suitable isolation method, structural and functional studies can now be performed to assess whether the fatty acid composition in EV-enriched fractions of human milk differs significantly from the whole human milk, and whether fatty acid composition of EVs relates to infant developmental outcomes. Future work could also consider whether maternal FADS genotype correlates to fatty acid composition in human milk and human milk EVs. 


\section{REFERENCES}

Albers, C. A., \& Grieve, A. J. (2007). Test Review: Bayley, N . ( 2006 ). "

Bayley Scales of Infant and Toddler Development -- Third Edition ". San Antonio, TX--Harcourt Assessment. Journal of Psychoeducational Assessment, 25, 180-190.

Angelsen, N. K., Vik, T., Jacobsen, G., \& Bakketeig, L. S. (2001). Breast feeding and cognitive development at age 1 and 5 years. Archives of Disease in Childhood, 85(3), 183-188.

Badr, L. K. (2009). Statistical Versus Clinical Significance for Infants With Brain Injury: Reanalysis of Outcome Data From a Randomized Controlled Study. Clinical Nursing Research, 18, 136-152.

Ballard, O., \& Morrow, A. L. (2013). Human milk composition: nutrients and bioactive factors. Pediatric Clinics of North America, 60, 49-74.

Barreiro, R., Díaz-Bao, M., Cepeda, A., Regal, P., \& Fente, C. A. (2018). Fatty acid composition of breast milk in Galicia (NW Spain): A cross-country comparison. Prostaglandins, Leukotrienes and Essential Fatty Acids, 135, 102-114.

Bayley, N. (2006). Bayley Scales of Infant and Toddler Development-

Third Edition. Journal of Psychoeducational

Assessment, 25(2), 180-198.

Berk, L. (2013). Child Development (9th ed.). Upper Saddle River, NJ: Pearson Education.

Bernard , J., Armand , M., Peyre , H., GARCIA , C., Forhan , A., De Agostini , M., . . Heude , B. (2017). Breastfeeding, Polyunsaturated Fatty Acid Levels in Colostrum and Child Intelligence Quotient at Age 5-6 Years. J Pediatr, 43-50.

Brzozowski, J. S., Jankowski, H., Bond, D. R., McCague, S. B., Munro, B. R., Predebon, M. J., . .. Weidenhofer, J. (2018). Lipidomic profiling of extracellular vesicles derived from prostate and prostate cancer cell lines. lipid in Health and Disease, 17, 211-223.

Caradec, J., Kharmate, G., Hosseini-Beheshti, E., Adomat, H., Gleave, M., \& Guns, E. (2014). Reproducibility and efficiency of serum-derived exosome extraction methods. Clinical Biochemistry, 47, 1286-1292.

Carlson, S. E., \& Colombo, J. (2016). Docosahexaenoic Acid and Arachidonic Acid Nutrition in Early Development. Advances in Pediatrics, 63, 453-471.

Carlson, S. E., Ford, A. J., Werkman, S. H., Peeples, J. M., \& Koo, W. W. K. (1996). Visual Acuity and Fatty Acid Status of Term Infants Fed Human Milk and Formulas with and without Docosahexaenoate and Arachidonate from Egg Yolk Lecithin. Nature, 39, 882888.

Carlson, S. E., Werkman, S. H., Peeples, J. M., Cooke, R. J., \& Tolley, E. A. (1993).

Arachidonic acid status correlates with first year growth in

preterm infants. Proceedings of the National Academy of Sciences of the United States of

America, 90(3).

CDC. (2018). Breastfeeding Report Card. Retrieved from https://www.cdc.gov/breastfeeding/data/reportcard.htm

Colombo, J., Shaddy, J., Kerling, E. H., Gustafson, K. M., \& Carlson, S. E. (2017). Docosahexaenoic acid (DHA) and arachidonic acid (ARA) balance in developmental outcomes. Prostaglandins, Leukotrienes and Essential Fatty Acids, 121, 52-56.

de Gassart, A., Geminard, C., Fevrier, B., Raposo, G., \& Vidal, M. (2003). Lipid-raft associated protein sorting in exosomes. Blood 103, 4336-4344. 
De la Torre Gomez, C., Goreham, R., Serra, J., Nann, T., \& Kussman, M. (2018). "Exosomics"A review of biophysics, biology and

biochemistry of exosomes with a focus on human breast milk. Frontiers in Genetics, 9, 1-11.

Demmelmair, H., Koletzko, B., \& panel, A. 1. o. o. (2018). Lipids in human milk. Best Practice \& Research Clinical Endocrinology \& Metabolism, 32, 57-68.

Food and Nutrition Board, I. o. M. (2002). Dietary Fats: Total Fat and Fatty Acids. Dietary Reference Intakes for Energy, Carbohydrate, Fiber, Fat, Fatty Acids, Cholesterol, Protein, and Amino Acids. 422-541.

Gallier, S., Vocking, K., Post, J. A., Van De Heijning, B., Acton, D., Van Der Beek, E., \& Baalen, T. V. (2015). A novel infant milk formula concept: Mimicking the human milk fat globule structure. Colloids and Surfaces B: Biointerfaces, 329-339.

Hadley, K. B., Ryan, A. S., Forsyth, S., Gautier, S., \& Salem, N. (2016). The Essentiality of Arachidonic Acid in Infant Development. Nutrients, 8.

Heaton, A. E., Meldrum, S. J., Foster, J. K., Prescott, S. L., \& Simmer, K. (2013). Does docosahexaenoic acid supplementation in term infants enhance neurocognitive functioning in infancy? Frontiers in Human Neuroscience.

Hock, A., Miyake, H., Li, B., Carol, L., Ermini, L., Koike, Y., . . Pierro, A. (2017a). Breast milk-derived exosomes promote intestinal epithelial cell growth. Journal of Pediatric Surgery, 52, 755-759.

Hock, A., Miyake, H., Li, B., Carol, L., Ermini, L., Koike, Y., . . Pierro, A. (2017b). Differences in exosome populations in human breast milk in relation to allergic sensitization and lifestyle. Journal of Pediatric Surgery, 52, 755-759.

Howard, K. M., Jati Kusuma, R., Baier, S. R., Friemel, T., Markham, L., Vanamala, J., \& Zempleni, J. (2015). Loss of miRNAs during processing and storage of cow's (Bos taurus) milk Journal of Agricultural Food Chemistry, 63, 588-592.

Janowska-Wieczorek, A., Wysoczynski, M., Kijowski, J., Marquez-Curtis, L., Machalinski, B., Ratajczak, J., \& Ratajczak, M. Z. (2005). Microvesicles derived from activated platelets induce metastasis and angiogenesis in lung cancer. International Journal of Cancer, 113, $752-760$.

Jensen, C. L., Voigt, R. G., Prager, T. C., Zou, Y. L., Fraley, J. K., Rozelle, J. C., . . Heird, W. C. (2005). Effects of maternal docosahexaenoic acid intake on visual function

and neurodevelopment in breastfed term infants. American Journal of Clinical Nutrition, 82, $125-132$.

Jeppesen, P. B., Høy, C. E., \& Mortensen, P. B. (1998). Essential fatty acid deficiency in patients receiving home parenteral nutrition. American Journal of Clinical Nutrition, 68, 126-133.

Kim, J., Tan, Z., \& Lubman, D. M. (2015). Exosome Enrichment of Human Serum using Multiple Cycles of

Centrifugation. Electrophoresis, 2017-2026.

Larque, E., Demmelmair, H., \& Koletzko, B. (2002). Perinatal Supply and Metabolism of LongChain Polyunsaturated Fatty Acids. Annals of The New York Academy of Sciences, 967, 299-310.

Lattka, E., Illig, T., Heinrich, J., \& Koletzko, B. (2009). FADS Gene Cluster Polymorphisms: Important Modulators of Fatty Acid Levels and Their Impact on Atopic Diseases. Journal of Nutrigenetics and Nutrigenomics, 2, 119-128.

Li, P., Kaslan, M., Lee, S. H., Yao, J., \& Gao, Z. (2017). Progress in Exosome Isolation Techniques. Theranostics, 7, 789-804. 
Lien, E. L., Richard, C., \& Hoffman, D. R. (2018). DHA and ARA addition to infant formula: Current status and future research directions. Prostaglandins, Leukotrienes, and Essential Fatty Acids 26-40.

Llorente, A., Skotland, T., Sylvänne, T., Kauhanen, D., Róg, T., Orlowski, A., . . Sandvig, K. (2013). Molecular lipidomics of exosomes released by PC-3 prostate cancer cells. Biochimica et Biophysica Acta (BBA) - Molecular and Cell Biology of Lipids, 1831, 1302-1309.

Makrides, M., Simmer, K., Goggin, M., \& Gibson, R. A. (1993). Erythrocyte Docosahexaenoic Acid Correlates

with the Visual Response of

Healthy, Term Infants. Pediatric Research, 33, 425-427.

Martin, C., Patel, M., Williams, S., Arora, H., \& Sims, B. (2018). Human breast milk derived exosomes attenuate cell death in intestinal epithelial cells. Innate Immunity, 24, 278-284.

Martin, M., Lassek, W., Gaulin, S., Evans, R., Woo, J., Geraghty, S., . . Gurven, M. (2012). Fatty acid composition in the mature milk of

Bolivian forager-horticulturalists: controlled comparisons with a US sample. Maternal and Child Nutrition, 8, 404-418.

Mimouni-Bloch, A., Kachevanskaya, A., Mimouni, F. B., Shuper, A., Raveh, E., \& Linder, N. (2013). Breastfeeding May Protect from Developing Attention-Deficit/Hyperactivity Disorder. Breastfeeding Medicine, 8.

Morley, R., Cole, T. J., Powell, R., \& Lucas, A. (1988). Mother's choice to provide breast milk and developmental outcome. Archives of Disease in Childhood, 63, 1382-1385.

Munagala, R., Aquil, F., Jeyabalan, J., \& Gupta, R. C. (2016). Bovine milk-derived exosomes for drug delivery. Cancer Letters, 371, 48-61.

Murphy, K. R., \& Myors, B. (2004). (Vol. 2): Taylor \& Francis.

Nakamura, M. T., \& Nara, T. Y. (2004). Structure, function, and dietary regulation of delta6, delta5, and delta9 desaturases. Annual Review of Nutrition, 24, 345-376.

Nielsen, M. H., Sabaratnam, R., James, A., Pedersen, T., Højlund, K., \& Handberg, A. (2019). Acute exercise increases plasma levels of muscle-derived microvesicles

carrying fatty acid transport proteins. The Journal of Clinical Endocrinology \& Metabolism.

Nommsen, L., Lovelady, C., Heinig, M., Lönnerdal, B., \& Dewey, K. (1991). Determinants of energy, protein, lipid, and lactose concentrations in human milk during the first 12 mo of lactation: the DARLING Study. American Journal of Clinical Nutrition, 53, 457-465.

Pang, W. W., Tan, P. T., Cai, S., Fok, D., Chua, M. C., Lim, S. B., . . Rifkin-Graboi, A. (2019). Nutrients or nursing? Understanding how breast milk feeding affects child cognition. European Journal of Nutrition, 1-11.

Pang, W. W., Tan, P. T., Cai, S., Fok, D., Chua, M. C., Lim, S. B., . . Tan, K. H., Yap, F., Gluckman, P.D. , Godfrey, K.M., Meaney, M.J., Broekmen, B.F.P., Kramer, M.S., Chong, Y., Rifkin-Graboi, A. (2020). Nutrients or nursing? Understanding how breast milk feeding affects child cognition. European Journal of Nutrition, 59, 608-619.

Pedersen, K. W., Kierulf, B., Oksvold, M. P., Li, M., Vlassov, A. V., Roos, N., . . Neurauter, A. (2015). Isolation and Characterization of Exosomes Using Magnetic Beads. BioProbes Journal of Cell Biology Applications, 71, 10-13. 
Samuel, M., Chisanga, D., Liem, M., Keerthikumar, S., Anand, S., Ang, C., . . Mathivanan, S. (2017). Bovine milk-derived exosomes from colostrum are enriched with proteins implicated in immune

response and growth. Scientific Reports, 7, 1-10.

SBI. (2018a). Exo-Check Exosome Antibody Arrays User Manual. Retrieved from https://www.systembio.com/wp-content/uploads/Exo-Check_Manual-1.pdf

SBI. (2018b). ExoQuick-TC® ULTRA EV Isolation Kit for

Tissue Culture Media User Manual. Retrieved from https:/www.systembio.com/wpcontent/uploads/MANUAL_ExoQuick-ULTRA_TC-1.pdf

SBI. (2018c). The Purest and Highest Yielding EV Isolation System. Retrieved from https://www.systembio.com/products/exosome-research/exosome-isolation/exoquickultra-for-serum-plasma/?gclid=Cj0KCQiAheXiBRD-ARIsAODSpWP8-yLL9-izE7IP9NPMHkhPoxQ_B7kFGrxoWXw7WYBa1915DdNDTEaAnKEEALw_wcB

Simbari, F. M., J. Coakley, G. Millar, M. Maizels, R.M. Fabrias, G. (2016). Plasmalogen enrichment in exosomes secreted by a nematode parasite versus those derived from its mouse host: implications for exosome stability and biology. Journal of Extracellular Vesicles, 5, 30741.

Skotland, T., Ekroos, K., Kauhanen, D., Simolin, H., Seierstad, T., Berge, V., . . Llorente, A. (2017). Molecular lipid species in urinary exosomes as potential prostate cancer

biomarkers. European Journal of Cancer, 70, 122-132.

Skotland, T., Sandvig, K., \& Llorente, A. (2017). Lipids in exosomes: Current knowledge and the

way forward. In (Vol. 66, pp. 30-41).

Sáenz-Cuesta, M., Arbelaiz, A., Oregi, A., Irizar, H., Osorio-Querejeta, I., Muñoz-Culla, M., . . . Otaegui, D. (2015). Methods for extracellular vesicles isolation in a hospital setting. Frontiers in Immunology, 6, 1-12.

ThermoFisher. (2018). Qubit TM 4 Fluorometer User Guide. Retrieved from https://assets.thermofisher.com/TFSAssets/LSG/manuals/MAN0017209_Qubit_4_Fluorometer_UG.pdf

Thery, C., Clayton, A., Amigorena, S., \& Raposo, G. (2006). Isolation and Characterization of Exosomes from Cell Culture Supernatants

and Biological Fluids. Current Protocols in Cell Biology, 30.

Théry, C., Witwer, K. W., \& Aikawa, E. (2018). Minimal information for studies of extracellular vesicles 2018 (MISEV2018): a position statement of the International Society for Extracellular Vesicles and update of the MISEV2014 guidelines. Journal of Extracellular Vesicles, 7(1).

Torregrosa Paredes, P., Gutzeit, C., Johansson, S., Admyre, C., Stenius, F., Alm, J., . . . Gabrielsson, S. (2014). Difference in exosome populations in human breast milk in relation to allergic sensitization and lifestyle. The European Journal of Allergy and Clinical Immunology, 69, 463-471.

Trajkovic, K., Hsu, C., Chiantia, S., Rajendran, L., Wenzel, D., Wieland, F., . . Simons, M. (2008). Ceramide Triggers Budding of Exosome Vesicles into Multivesicular Endosomes. Science, 319, 1244-1247.

Tressou, J., Buaud, B., Simon, N., Pasteau, S., \& Guesnet, P. (2019). Very low inadequate dietary intakes of essential n-3 polyunsaturated fatty acids (PUFA) in pregnant and 
lactating French women: The INCA2 survey. Prostaglandins, Leukotrienes, and Essential Fatty Acids, 140, 3-10.

Valentine, C., Morrow, G., Pennell, M., Morrow, A., Hodge, A., Haban-Bartz, . . Rogers, L. (2012). Randomized

Controlled Trial of Docosahexaenoic Acid Supplementation in Midwestern U.S.

Human Milk Donors. Breastfeeding Medicine, 8, 86-91.

Van der Pol, E., Hoekstra, A. G., Sturk, A., Otto, C., van Leeuwen, T. G., \& Nieuwland, R. (2010). Optical and non-optical

methods for detection and characterization of microparticles and exosomes. Journal of

Thrombosis and Haemostasis 2596 - 2607. doi:10.1111/j.1538-7836.2010.04074.x.

van Goor, S., Dijck-Brouwer, D. A. J., Doornbos, B., Erwich, J. J. H. M., Schaafsma, A., Muskiet, F. A. J., \& Hadders-Algra, M. (2010). Supplementation of DHA but not DHA with arachidonic acid during pregnancy and lactation influences general movement quality in 12-week-old term infants. British Journal of Nutrition, 103, 235-242.

Vaswani, K., Qin Koh, Y., Almughlliq, F. B., Peiris, H. N., \& Mitchell, M. D. (2017). A method for the isolation and enrichment of purified bovine milk exosomes. Reproductive Biology, 17, 341-348.

Vergilio Visentainer, J., Oliveira Santos, O., Maldaner, L., Zappielo, C., Neia, V., Visentainer, L., . . Laguila Visentainer, J. (2018). Lipids and Fatty Acids in Human Milk: Benefits and Analysis. In Biochemistry and Health Benefits of Fatty Acids

Walfisch, A., Sermer, C., Cressman, A., \& Koren, G. (2013). Breast milk and cognitive development - the role of confounders: a systematic review. BMJ Open, 3.

Wang, X. (2017). Extracellular Vesicles: Methods and Protocols (W. P. Kuo \& S. Jia Eds. Vol. Journal of Extracellular Vesicles). New York: Springer.

Wang, X. (2017). Isolation of Extracellular Vesicles from Breast Milk (Vol. Journal of Extracellular Vesicles).

WHO. (2013). Definition of Key Terms. Retrieved from https://www.who.int/hiv/pub/guidelines/arv2013/intro/keyterms/en/

Yamada, T., Inoshima, Y., Matsuda, T., \& Ishiguro, N. (2012). Comparison of Methods for Isolating Exosomes from Bovine Milk. Journal of Veterinary Medicine, 74, 1523-1525.

Yang, M., Deng, W., Cao, X., Wang, L., Yu, N., Zheng, Y., . . Yue, X. (2020). Quantitative Phosphoproteomics of Milk Fat Globule Membrane in Human Colostrum and Mature Milk: New Insights into Changes in Protein Phosphorylation during Lactation. Journal of Agricultural and Food Chemistry, 68, 4546-4556.

Zonneveld, M., Brisson, A., van Herwijnen, M., Tan, S., van de Lest, C., Redegeld, F., . . . Nolte-'t Hoen, E. (2014). Recovery of extracellular vesicles from human breast milk is influenced by sample collection and vesicle isolation procedures. Journal of Extracellular Vesicles, 3. 